\title{
Algebraic Approach to Algorithmic Logic
}

\author{
Grzegorz Bancerek \\ Association of Mizar Users \\ Białystok, Poland
}

\begin{abstract}
Summary. We introduce algorithmic logic - an algebraic approach according to 25. It is done in three stages: propositional calculus, quantifier calculus with equality, and finally proper algorithmic logic. For each stage appropriate signature and theory are defined. Propositional calculus and quantifier calculus with equality are explored according to 24]. A language is introduced with language signature including free variables, substitution, and equality. Algorithmic logic requires a bialgebra structure which is an extension of language signature and program algebra. While-if algebra of generator set and algebraic signature is bialgebra with appropriate properties and is used as basic type of algebraic logic.
\end{abstract}

MSC: 03B05 03B10 03B35

Keywords: propsitional calcus; quantifier calcus; algorithmic logic

MML identifier: AOFA_L00, version: 8.1.03 5.25.1220

The notation and terminology used in this paper have been introduced in the following articles: [1, [2], [15], 3], [4, [5, [12], [16], 22], 26], 29], [17, [18], [13], 6], 34], 27], 31], 9], 20], 32], 35], 33], 23], [14, and [21].

\section{Algorithmic Langugage Signature}

From now on $\mathcal{X}, Y$ denote sets, $x, y, z$ denote objects, and $i, j, n$ denote natural numbers.

Let $f$ be a non empty yielding function. One can check that $\bigcup f$ is non empty.

Let $I$ be a set, $f$ be a many sorted set indexed by $I$, and $i$ be a set. Let us consider $x$. One can verify that the functor $f+\cdot(i, x)$ yields a many sorted set indexed by $I$. Let $f$ be a non-empty many sorted set indexed by $I$ and $x$ be a non empty set. One can check that $f+\cdot(i, x)$ is non-empty. 
Let $\Sigma$ be a non empty, non void many sorted signature. Let us observe that there exists a strict free variable algebra over $\Sigma$ which is non-empty.

Let $f, g$ be functions. We say that $g$ is $f$-tolerating if and only if (Def. 1) $f \approx g$.

Now we state the propositions:

(1) Let us consider functions $f, g$. Then $g$ is $f$-tolerating if and only if for every $x$ such that $x \in \operatorname{dom} f$ and $x \in \operatorname{dom} g$ holds $f(x)=g(x)$.

(2) Let us consider sets $I, \Omega$, a many sorted set $f$ indexed by $I$, and a many sorted set $g$ indexed by $\Omega$. Then $g$ is $f$-tolerating if and only if for every $x$ such that $x \in I$ and $x \in \Omega$ holds $f(x)=g(x)$. The theorem is a consequence of (1).

(3) Let us consider functions $f, g$. Then $f \approx g+\cdot f$.

Let $\mathcal{X}, Y$ be functions. Note that $Y+\cdot \mathcal{X}$ is $\mathcal{X}$-tolerating.

Let $\mathcal{X}$ be a function, $\Omega$ be a set, and $Y$ be a many sorted set indexed by $\Omega$. Observe that $Y+\cdot \mathcal{X} \backslash \Omega$ is $\mathcal{X}$-tolerating and there exists a many sorted set indexed by $\Omega$ which is $\mathcal{X}$-tolerating.

Let $\mathcal{X}$ be a non-empty function. Note that there exists a non-empty many sorted set indexed by $\Omega$ which is $\mathcal{X}$-tolerating.

Let $I$ be a non empty set and $\mathcal{X}$ be a non empty yielding many sorted set indexed by $I$. One can verify that $\bigcup \mathcal{X}$ is non empty.

Now we state the propositions:

(4) Let us consider a non empty, non void many sorted signature $\Sigma$, an operation symbol $o$ of $\Sigma$, a sort symbol $r$ of $\Sigma$, and an algebra $\mathfrak{T}$ over $\Sigma$. If $o$ is of type $\emptyset \rightarrow r$, then $\emptyset \in \operatorname{Args}(o, \mathfrak{T})$.

(5) Let us consider a non empty, non void many sorted signature $\Sigma$, an operation symbol $o$ of $\Sigma$, sort symbols $\sigma, r$ of $\Sigma$, and an algebra $\mathfrak{T}$ over $\Sigma$. Suppose $o$ is of type $\langle\sigma\rangle \rightarrow r$ and $x \in($ the sorts of $\mathfrak{T})(\sigma)$. Then $\langle x\rangle \in$ $\operatorname{Args}(o, \mathfrak{T})$.

(6) Let us consider a non empty, non void many sorted signature $\Sigma$, an operation symbol $o$ of $\Sigma$, sort symbols $\sigma_{1}, \sigma_{2}, r$ of $\Sigma$, and an algebra $\mathfrak{T}$ over $\Sigma$. Suppose $o$ is of type $\left\langle\sigma_{1}, \sigma_{2}\right\rangle \rightarrow r$ and $x \in$ (the sorts of $\left.\mathfrak{T}\right)\left(\sigma_{1}\right)$ and $y \in($ the sorts of $\mathfrak{T})\left(\sigma_{2}\right)$. Then $\langle x, y\rangle \in \operatorname{Args}(o, \mathfrak{T})$.

(7) Let us consider a non empty, non void many sorted signature $\Sigma$, an operation symbol $o$ of $\Sigma$, sort symbols $\sigma_{1}, \sigma_{2}, \sigma_{3}, r$ of $\Sigma$, and an algebra $\mathfrak{T}$ over $\Sigma$. Suppose $o$ is of type $\left\langle\sigma_{1}, \sigma_{2}, \sigma_{3}\right\rangle \rightarrow r$ and $x \in$ (the sorts of $\mathfrak{T})\left(\sigma_{1}\right)$ and $y \in($ the sorts of $\mathfrak{T})\left(\sigma_{2}\right)$ and $z \in($ the sorts of $\mathfrak{T})\left(\sigma_{3}\right)$. Then $\langle x$, $y, z\rangle \in \operatorname{Args}(o, \mathfrak{T})$.

Let $\Sigma, E$ be signatures. We say that $E$ is $\Sigma$-extension if and only if 
(Def. 2) $\Sigma$ is a subsignature of $E$.

Let $\Sigma$ be a signature. Let us observe that every extension of $\Sigma$ is $\Sigma$-extension. Now we state the propositions:

(8) Let us consider non empty signatures $\Sigma$, $E$. Suppose $E$ is $\Sigma$-extension. Then every sort symbol of $\Sigma$ is a sort symbol of $E$.

(9) Let us consider non void signatures $\Sigma, E$. Suppose $E$ is $\Sigma$-extension. Let us consider an operation symbol $o$ of $\Sigma$, a set $a$, an element $r$ of $\Sigma$, and an element $r_{1}$ of $E$. If $r=r_{1}$ and $o$ is of type $a \rightarrow r$, then $o$ is of type $a$ $\rightarrow r_{1}$.

Let $\mathcal{X}$ be a function and $\Omega, Y$ be sets. The functor $\mathcal{X}$ extended by $(Y, \Omega)$ yielding a many sorted set indexed by $\Omega$ is defined by the term

(Def. 3) $(\Omega \longmapsto Y)+\cdot \mathcal{X}\lceil\Omega$.

One can check that $\mathcal{X}$ extended by $(Y, \Omega)$ is $\mathcal{X}$-tolerating.

We consider PC language signatures which extend connectives signatures and are systems

〈a carrier, a carrier', an arity, a result sort,

a formula sort, connectives $\rangle$

where the carrier is a set, the carrier' is a set, the arity is a function from the carrier' into (the carrier) ${ }^{*}$, the result sort is a function from the carrier' into the carrier, the formula sort is an element of the carrier, the connectives constitute a finite sequence of elements of the carrier'.

Let $\mathcal{X}$ be a set. We consider $\mathrm{QC}$ language signatures over $\mathcal{X}$ which extend $\mathrm{PC}$ language signatures and are systems

〈a carrier, a carrier', an arity, a result sort,

a formula sort, connectives, a quantifier sort, quantifiers

where the carrier is a set, the carrier' is a set, the arity is a function from the carrier' into (the carrier) ${ }^{*}$, the result sort is a function from the carrier' into the carrier, the formula sort is an element of the carrier, the connectives constitute a finite sequence of elements of the carrier', the quantifier sort is a set, the quantifiers constitute a function from (the quantifier sort) $\times \mathcal{X}$ into the carrier'.

We consider algorithmic language signatures of $\mathcal{X}$ which extend QC language signatures over $\mathcal{X}$ and are systems

〈a carrier, a carrier', an arity, a result sort,

a formula sort, a program sort, connectives, a quantifier sort, quantifiers 
where the carrier is a set, the carrier' is a set, the arity is a function from the carrier' into (the carrier) ${ }^{*}$, the result sort is a function from the carrier' into the carrier, the formula sort and the program sort are elements of the carrier, the connectives constitute a finite sequence of elements of the carrier', the quantifier sort is a set, the quantifiers constitute a function from (the quantifier sort) $\times \mathcal{X}$ into the carrier'.

Let $n$ be a natural number and $L$ be a PC language signature. We say that $L$ is PC correct w.r.t. $n$ if and only if

(Def. 4) len(the connectives of $L) \geqslant n+5$ and (the connectives of $L)\lceil\{n, n+1, n+$ $2, n+3, n+4, n+5\}$ is one-to-one and (the connectives of $L)(n)$ is of type $\langle$ the formula sort of $L\rangle \rightarrow$ the formula sort of $L$ and (the connectives of $L)(n+5)$ is of type $\emptyset \rightarrow$ the formula sort of $L$ and (the connectives of $L)(n+1)$ is of type $\langle$ the formula sort of $L$, the formula sort of $L\rangle \rightarrow$ the formula sort of $L$ and $\ldots$ and (the connectives of $L)(n+4)$ is of type $\langle$ the formula sort of $L$, the formula sort of $L\rangle \rightarrow$ the formula sort of $L$.

Let us consider $\mathcal{X}$. Let $L$ be a $\mathrm{QC}$ language signature over $\mathcal{X}$. We say that $L$ is QC correct if and only if

(Def. 5) the quantifier sort of $L=\{1,2\}$ and the quantifiers of $L$ is one-to-one and $\operatorname{rng}($ the quantifiers of $L$ ) misses $\operatorname{rng}($ the connectives of $L$ ) and for every objects $q, x$ such that $q \in$ the quantifier sort of $L$ and $x \in \mathcal{X}$ holds (the quantifiers of $L)(q, x)$ is of type $\langle$ the formula sort of $L\rangle \rightarrow$ the formula sort of $L$.

Let $n$ be a natural number, $\mathcal{X}$ be a set, and $L$ be an algorithmic language signature of $\mathcal{X}$. We say that $L$ is AL correct w.r.t. $n$ if and only if

(Def. 6) the program sort of $L \neq$ the formula sort of $L$ and len(the connectives of $L) \geqslant n+8$ and (the connectives of $L)(n+6$ ) is of type 〈the program sort of $L$, the formula sort of $L\rangle \rightarrow$ the formula sort of $L$ and ... and (the connectives of $L)(n+8)$ is of type <the program sort of $L$, the formula sort of $L\rangle \rightarrow$ the formula sort of $L$.

Let us consider $n$. Let us note that every PC language signature which is PC correct w.r.t. $n$ is also non void.

Let $\mathcal{X}, Y$ be sets. Assume $Y \subseteq \mathcal{X}$. The functor $\operatorname{incl}(Y, \mathcal{X})$ yielding a function from $Y$ into $\mathcal{X}$ is defined by the term

(Def. 7) $\operatorname{id}_{Y}$.

Let $n$ be a non empty, natural number and $\mathcal{X}$ be a set. Let us observe that there exists a QC language signature over $\mathcal{X}$ which is non void, non empty, PC correct w.r.t. $n$, and $\mathrm{QC}$ correct and there exists a $\mathrm{PC}$ language signature which is non void, non empty, and $\mathrm{PC}$ correct w.r.t. $n$.

Let $\mathcal{X}$ be a set. Note that there exists a strict algorithmic language signature 
of $\mathcal{X}$ which is non void and non empty and every set which is ordinal is also non pair.

(10) Let us consider an ordinal number $a$ and natural numbers $n_{1}, n_{2}$. If $n_{1} \neq n_{2}$, then $a+n_{1} \neq a+n_{2}$.

Let $R$ be a non empty binary relation. Observe that every element of $R$ is pair.

(11) Let us consider a non empty, natural number $n$, a non empty set $\mathcal{X}$, and a signature $\Omega$. Then there exists a strict, non void, non empty algorithmic language signature $\Sigma$ of $\mathcal{X}$ such that

(i) $\Sigma$ is $\mathrm{PC}$ correct w.r.t. $n$, QC correct, $\mathrm{AL}$ correct w.r.t. $n$, and $\Omega$ extension, and

(ii) for every $i$ such that $i=0$ or $\ldots$ or $i=8$ holds (the connectives of $\Sigma)(n+i)=\sup ($ the carrier' of $\Omega)+i$, and

(iii) for every element $x$ of $\mathcal{X}$, (the quantifiers of $\Sigma)(1, x)=\langle$ the carrier' of $\Omega, 1, x\rangle$ and (the quantifiers of $\Sigma)(2, x)=\langle$ the carrier' of $\Omega, 2, x\rangle$, and

(iv) the formula sort of $\Sigma=\sup ($ the carrier of $\Omega$ ), and

(v) the program sort of $\Sigma=\sup ($ the carrier of $\Omega)+1$, and

(vi) the carrier of $\Sigma=($ the carrier of $\Omega) \cup\{$ the formula sort of $\Sigma$, the program sort of $\Sigma$ \}, and

(vii) for every ordinal number $w$ such that $w=\sup ($ the carrier' of $\Omega$ ) holds the carrier' of $\Sigma=(($ the carrier' of $\Omega) \cup\{w+0, w+1, w+2, w+3, w+$ $4, w+5, w+6, w+7, w+8\}) \cup(\{$ the carrier' of $\Omega\} \times\{1,2\} \times \mathcal{X})$.

Proof: Set $w=\sup ($ the carrier' of $\Omega$ ). Set $u=\sup ($ the carrier of $\Omega$ ). Set $O_{1}=\{w+0, w+1, w+2, w+3, w+4, w+5, w+6, w+7, w+8\} \cup(\{$ the carrier' of $\Omega\} \times\{1,2\} \times \mathcal{X})$. Set $O=($ the carrier' of $\Omega) \cup O_{1}$. Set $a=(((\{w+$ $1, w+2, w+3, w+4\} \longmapsto\langle u+0, u+0\rangle) \cup(\{w+0\} \cup(\{$ the carrier' of $\Omega\} \times$ $\{1,2\} \times \mathcal{X}) \longmapsto\langle u+0\rangle)) \cup(\{w+5\} \longmapsto \emptyset)) \cup(\{w+6, w+7, w+8\} \longmapsto$ $\langle u+1, u+0\rangle)$. Set $r=O_{1} \longmapsto u+0$. Set $r_{2}=$ (the result sort of $\Omega)+\cdot r .\{w+0, w+1, w+2, w+3, w+4, w+5, w+6, w+7, w+8\}$ misses $\{$ the carrier' of $\Omega\} \times\{1,2\} \times \mathcal{X}$ by [30, (68)]. $\{w+1, w+2, w+3, w+4\}$ misses $\{w+0\} \cup(\{$ the carrier' of $\Omega\} \times\{1,2\} \times \mathcal{X})$ by [11, (21)], [30, (68)]. $\{w+0, w+1, w+2, w+3, w+4\} \cup(\{$ the carrier' of $\Omega\} \times\{1,2\} \times \mathcal{X})$ misses $\{w+5\}$ by [11, (21)], [30, (68)]. $\{w+0, w+1, w+2, w+3, w+$ $4, w+5\} \cup(\{$ the carrier' of $\Omega\} \times\{1,2\} \times \mathcal{X})$ misses $\{w+6, w+7, w+8\}$ by [11, (21)], [30, (68)]. $O_{1}$ misses the carrier' of $\Omega$ by [10, (19), (27)], [30, (68)], [11, (22)]. Reconsider $a_{1}=$ (the arity of $\Omega$ ) $\cup a$ as a function. Set $\gamma=($ the carrier of $\Omega) \cup\{u+0, u+1\}$. Reconsider $0_{0}=u+0,0_{1}=u+1$ as 
an element of $\gamma$. Reconsider $o_{0}=w+0, o_{1}=w+1, o_{2}=w+2, o_{3}=w+3$, $o_{4}=w+4, o_{5}=w+5, o_{6}=w+6, o_{7}=w+7, o_{8}=w+8$ as an element of $O$. Set $p=$ the $\left(n-{ }^{\prime} 1\right.$ qua natural number $)$-element finite sequence of elements of $\{w+0, w+1, w+2, w+3, w+4, w+5, w+6, w+7, w+8\}$. Set $c^{\prime}=\left\langle o_{0}, o_{1}, o_{2}, o_{3}, o_{4}, o_{5}, o_{6}, o_{7}\right\rangle \frown\left\langle o_{8}\right\rangle$. Reconsider $c=p^{\frown c^{\prime}}$ as a finite sequence of elements of $O$. If $i=0$ or $\ldots$ or $i=8$, then $c(n+i)=w+i$ by [2, (29)]. Define $\mathcal{Q}$ (object) $=\left\langle\right.$ the carrier' of $\left.\Omega, \$_{11}, \$_{12}\right\rangle$. Consider $q$ being a function such that $\operatorname{dom} q=\{1,2\} \times \mathcal{X}$ and for every $x$ such that $x \in\{1,2\} \times$ $\mathcal{X}$ holds $q(x)=\mathcal{Q}(x)$ from [17, Sch. 3]. $\operatorname{rng} q \subseteq O$ by [30, (69)]. Set $L=$ $\left\langle\gamma, O, a_{1}, r_{2}, 0_{0}, 0_{1}, c,\{1,2\}, q\right\rangle$. Set $N=\{n, n+1, n+2, n+3, n+4, n+5\}$. (The connectives of $L)\lceil N$ is one-to-one by [35, (57)], [17, (49)], [11, (21)]. (the connectives of $L)(n)$ is of type $\langle$ the formula sort of $L\rangle \rightarrow$ the formula sort of $L$ by [21, (106)], [17, (1)], [21, (87)], [19, (13)]. (the connectives of $L)(n+5)$ is of type $\emptyset \rightarrow$ the formula sort of $L$ by [21, (106)], [17, (1)], [21, (87)], [19, (13)]. (the connectives of $L)(n+1)$ is of type / the formula sort of $L$, the formula sort of $L\rangle \rightarrow$ the formula sort of $L$ and $\ldots$ and (the connectives of $L)(n+4)$ is of type <the formula sort of $L$, the formula sort of $L\rangle \rightarrow$ the formula sort of $L$ by [21, (106)], [17, (1)], [19, (13)]. The quantifiers of $L$ is one-to-one. $\operatorname{rng}($ the quantifiers of $L) \subseteq\{$ the carrier' of $\Omega\} \times\{1,2\} \times \mathcal{X}$ by [30, (10), (69)]. (the connectives of $L)(n+6)$ is of type $\langle$ the program sort of $L$, the formula sort of $L\rangle \rightarrow$ the formula sort of $L$ and $\ldots$ and (the connectives of $L)(n+8)$ is of type / the program sort of $L$, the formula sort of $L\rangle \rightarrow$ the formula sort of $L$ by [21, (106)], [17, (1)], [19, (13)]. $L$ is $\Omega$-extension by [35, (53)], [19, (33)], [35, (65)], [17, (102)].

Let $n$ be a non empty, natural number, $\mathcal{X}$ be a non empty set, and $\Omega$ be a signature. Let us observe that there exists a non void, non empty, strict algorithmic language signature of $\mathcal{X}$ which is $\Omega$-extension, $\mathrm{PC}$ correct w.r.t. $n$, $\mathrm{QC}$ correct, and AL correct w.r.t. $n$ and there exists a non void, non empty, strict algorithmic language signature of $\mathcal{X}$ which is $\mathrm{PC}$ correct w.r.t. $n$, $\mathrm{QC}$ correct, and AL correct w.r.t. $n$.

\section{LANGUAGE}

Let $\Omega$ be a non empty, non void signature and $\mathfrak{T}$ be an algebra over $\Omega$.

A variable set in $\mathfrak{T}$ is a set and is defined by

(Def. 8) there exists a generator set $G$ of $\mathfrak{T}$ such that $i t=\bigcup G$.

Let $\mathcal{X}$ be a generator set of $\mathfrak{T}$. Note that the functor $\bigcup \mathcal{X}$ yields a variable set in $\mathfrak{T}$. Now we state the proposition: 
(12) Let us consider a non empty, non void signature $\Omega$, an algebra $\mathfrak{T}$ over $\Omega$, and a variable set $\mathcal{X}$ in $\mathfrak{T}$. Then $\mathcal{X} \subseteq \bigcup($ the sorts of $\mathfrak{T})$.

Let $\Sigma$ be a non empty, non void signature, $\mathcal{X}$ be a many sorted set indexed by the carrier of $\Sigma$, and $\mathfrak{T}$ be a free variable algebra over $\Sigma$. We say that $\mathfrak{T}$ is $\mathcal{X}$-vf-yielding if and only if

(Def. 9) the free variables of $\mathfrak{T}$ is a double many sorted set of the sorts of $\mathfrak{T}$ and $\mathcal{X}$.

Let $\Omega$ be a non empty set, $Q$ be a many sorted set indexed by $\Omega, Y$ be a set, and $f$ be a function from $\cup Q \times Y$ into $\cup Q$. We say that $f$ is sort-preserving if and only if

(Def. 10) for every element $j$ of $\Omega, f^{\circ}(Q(j) \times Y) \subseteq Q(j)$.

One can verify that there exists a function from $\cup Q \times Y$ into $\bigcup Q$ which is sort-preserving.

Let $\Omega$ be a non empty, non void signature and $\mathcal{X}$ be a many sorted set indexed by the carrier of $\Omega$. We consider algebras over $\Omega, \mathcal{X}$ with substitution which extend algebras over $\Omega$ and are systems

\section{〈sorts, a characteristics, a substitution operation〉}

where the sorts constitute a many sorted set indexed by the carrier of $\Omega$, the characteristics is a many sorted function from (the sorts) \# ( the arity of $\Omega$ ) into (the sorts) $\cdot($ the result sort of $\Omega$ ), the substitution operation is a sort-preserving

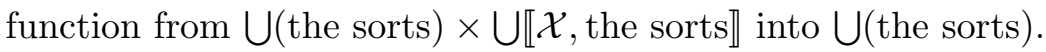

Now we state the proposition:

(13) Let us consider a set $I$, a many sorted set $\mathcal{X}$ indexed by $I$, a many sorted subset $\Sigma$ of $\mathcal{X}$, and $x$. Then $\Sigma(x)$ is a subset of $\mathcal{X}(x)$.

Let $\Omega$ be a non empty, non void signature, $\mathcal{X}$ be a non empty yielding many sorted set indexed by the carrier of $\Omega$, and $Q$ be an algebra over $\Omega, \mathcal{X}$ with substitution. Assume $\mathcal{X}$ is a many sorted subset of the sorts of $Q$. Let $x$ be an element of $\bigcup \mathcal{X}$. The functor ${ }^{\circledR}(x, Q)$ yielding an element of $\bigcup$ (the sorts of $\left.Q\right)$ is defined by the term

(Def. 11) $x$.

Assume $\mathcal{X}$ is a many sorted subset of the sorts of $Q$. Let $j$ be a sort symbol of $\Omega$. Assume (the sorts of $Q)(j) \neq \emptyset$. Let $\alpha$ be an element of $Q$ from $j$ and $y$ be an element of $\bigcup \mathcal{X}$. Given sort symbol $a$ of $\Omega$ such that $x, y \in \mathcal{X}(a)$. The functor $\alpha_{(x \leftarrow y)}$ yielding an element of $Q$ from $j$ is defined by the term

(Def. 12) (the substitution operation of $Q)(\langle\alpha,\langle x, y\rangle\rangle)$.

Assume (the sorts of $Q)(j) \neq \emptyset$. Let $\tau$ be an element of $\bigcup($ the sorts of $Q$ ). Given sort symbol $a$ of $\Omega$ such that $x \in \mathcal{X}(a)$ and $\tau \in($ the sorts of $Q)(a)$. The functor $\alpha_{(x \leftarrow \tau)}$ yielding an element of $Q$ from $j$ is defined by the term 
(Def. 13) (the substitution operation of $Q)(\langle\alpha,\langle x, \tau\rangle\rangle)$.

Let $\Omega$ be a non empty, non void many sorted signature and $\mathcal{X}$ be a many sorted set indexed by the carrier of $\Omega$. One can verify that there exists an algebra over $\Omega, \mathcal{X}$ with substitution which is non-empty.

Let $\Omega$ be a non empty, non void signature, $\mathcal{X}$ be a non empty yielding many sorted set indexed by the carrier of $\Omega, Q$ be a non-empty algebra over $\Omega, \mathcal{X}$ with substitution, $o$ be an operation symbol of $\Omega, p$ be an element of $\operatorname{Args}(o, Q), x$ be an element of $\bigcup \mathcal{X}$, and $y$ be an element of $\bigcup$ (the sorts of $Q$ ). The functor $p_{(x \leftarrow y)}$ yielding an element of $\operatorname{Args}(o, Q)$ is defined by

(Def. 14) for every natural number $i$ such that $i \in \operatorname{dom} \operatorname{Arity}(o)$ there exists a sort symbol $j$ of $\Omega$ such that $j=\operatorname{Arity}(o)(i)$ and there exists an element $\alpha$ of $Q$ from $j$ such that $\alpha=p(i)$ and $i t(i)=\alpha_{(x \leftarrow y)}$.

Let $I$ be a non empty set, $\mathcal{X}$ be a non-empty many sorted set indexed by $I$, $\Sigma$ be a non-empty many sorted subset of $\mathcal{X}, x$ be an element of $I$, and $z$ be an element of $\Sigma(x)$. The functor ${ }^{{ }} z$ yielding an element of $\mathcal{X}(x)$ is defined by the term

(Def. 15) z.

Let $\Omega$ be a non empty, non void signature, $\mathcal{X}$ be a non empty yielding many sorted set indexed by the carrier of $\Omega$, and $Q$ be a non-empty algebra over $\Omega$, $\mathcal{X}$ with substitution. We say that $Q$ is substitution correct (1) if and only if

(Def. 16) for every element $x$ of $\bigcup \mathcal{X}$ and for every sort symbol $a$ of $\Omega$ such that $x \in \mathcal{X}(a)$ holds for every sort symbol $j$ of $\Omega$ and for every element $\alpha$ of $Q$ from $j, \alpha_{(x \leftarrow x)}=\alpha$ and for every element $y$ of $\bigcup($ the sorts of $Q)$ such that $y \in($ the sorts of $Q)(a)$ for every operation symbol $o$ of $\Omega$ for every element $p$ of $\operatorname{Args}(o, Q)$ for every element $\alpha$ of $Q$ from the result sort of $o$ such that $\alpha=(\operatorname{Den}(o, Q))(p)$ holds if there exists no QC language signature $\Sigma$ over $\bigcup \mathcal{X}$ such that $\Omega=\Sigma$ and there exists an element $z$ of $\bigcup \mathcal{X}$ and there exists an element $q$ of $\{1,2\}$ such that $o=($ the quantifiers of $\Sigma)(q, z)$, then $\alpha_{(x \leftarrow y)}=(\operatorname{Den}(o, Q))\left(p_{(x \leftarrow y)}\right)$.

We say that $Q$ is substitution correct (2) if and only if

(Def. 17) for every sort symbol $j$ of $\Omega$ and for every element $q$ of $Q$ from $j$ and for every element $\tau$ of $Q$ from $j$ and for every element $x$ of $\cup \mathcal{X}$ such that $\tau=x \in \mathcal{X}(j)$ holds $\tau_{(x \leftarrow q)}=q$.

Now we state the proposition:

(14) Let us consider a non empty, non void signature $\Omega$, a non empty yielding many sorted set $\mathcal{X}$ indexed by the carrier of $\Omega$, and an algebra $Q$ over $\Omega$, $\mathcal{X}$ with substitution. Suppose $\mathcal{X}$ is a many sorted subset of the sorts of $Q$. Let us consider a sort symbol $a$ of $\Omega$. Suppose (the sorts of $Q)(a) \neq \emptyset$. Let us consider an element $\alpha$ of $Q$ from $a$, elements $x, y$ of $\cup \mathcal{X}$, and an 
element $\tau$ of $\bigcup($ the sorts of $Q)$. Suppose $y=\tau$. Let us consider a sort symbol $j$ of $\Omega$. If $x, y \in \mathcal{X}(j)$, then $\alpha_{(x \leftarrow y)}=\alpha_{(x \leftarrow \tau)}$. The theorem is a consequence of (13).

Let $\Omega$ be a non void signature. Let us note that every signature which is $\Omega$-extension is also non void and non empty.

Let $\Omega$ be a signature. Let us note that there exists a non empty, non void signature which is $\Omega$-extension.

Let $\mathcal{X}$ be a non empty set. Let us note that there exists a non empty, non void QC language signature over $\mathcal{X}$ which is $\Omega$-extension.

Let $n$ be a non empty, natural number. Note that there exists a non empty, non void, PC correct w.r.t. $n$, QC correct QC language signature over $\mathcal{X}$ which is $\Omega$-extension.

Let $n$ be a non empty natural number and $\Sigma$ be an $\Omega$-extension, PC correct w.r.t. $n$, feasible algorithmic language signature of $\mathcal{X}$. We say that $\Sigma$ is essential if and only if

(Def. 18) (the connectives of $\Sigma)^{\circ}(n+9 \backslash n)$ misses the carrier' of $\Omega$ and $\operatorname{rng}($ the quantifiers of $\Sigma$ ) misses the carrier' of $\Omega$ and the formula sort of $\Sigma$, the program sort of $\Sigma$ \} misses the carrier of $\Omega$.

Let $n$ be a non empty, natural number. Observe that there exists an $\Omega$ extension, PC correct w.r.t. $n$, QC correct, $\mathrm{AL}$ correct w.r.t. $n$, non void, non empty, strict algorithmic language signature of $\mathcal{X}$ which is essential.

Let $\Omega$ be a non empty signature, $\Sigma$ be an $\Omega$-extension, non empty signature, and $\mathcal{X}$ be a non empty yielding many sorted set indexed by the carrier of $\Omega$. Note that there exists a non empty yielding many sorted set indexed by the carrier of $\Sigma$ which is $\mathcal{X}$-tolerating.

Let $\Sigma$ be a non empty signature, $\mathcal{T}$ be an algebra over $\Omega$, and $Q$ be an algebra over $\Sigma$. We say that $Q$ is $\mathfrak{T}$-extension if and only if

(Def. 19) $Q \uparrow \Omega=$ the algebra of $\mathfrak{T}$.

Now we state the propositions:

(15) Let us consider a non empty, non void signature $\Omega$, an $\Omega$-extension signature $\Sigma$, an algebra $\mathfrak{T}$ over $\Omega$, and algebras $Q_{1}, Q_{2}$ over $\Sigma$. Suppose the algebra of $Q_{1}=$ the algebra of $Q_{2}$. If $Q_{1}$ is $\mathfrak{T}$-extension, then $Q_{2}$ is T-extension.

(16) Let us consider a non empty, non void signature $\Omega$, an $\Omega$-extension signature $\Sigma$, an algebra $\mathfrak{T}$ over $\Omega$, and an algebra $Q$ over $\Sigma$. Suppose $Q$ is $\mathfrak{T}$-extension. Suppose $x \in$ the carrier of $\Omega$. Then (the sorts of $\mathfrak{T})(x)=($ the sorts of $Q)(x)$.

(17) Let us consider a non empty, non void signature $\Omega$, an $\Omega$-extension signature $\Sigma$, an algebra $\mathfrak{T}$ over $\Omega$, and a set $I$. Suppose $I \subseteq$ (the carrier of 
$\Sigma) \backslash($ the carrier of $\Omega$ ). Let us consider a many sorted set $\mathcal{X}$ indexed by $I$. Then there exists an algebra $Q$ over $\Sigma$ such that

(i) $Q$ is $\mathfrak{T}$-extension, and

(ii) (the sorts of $Q)\lceil I=\mathcal{X}$.

Proof: Set $\mathfrak{U}=$ (the many sorted set indexed by the carrier of $\Sigma+\cdot$ (the sorts of $\mathfrak{T}))+\cdot \mathcal{X}$. Set $\gamma=$ the many sorted function from $\mathfrak{U}^{\#} \cdot($ the arity of $\Sigma$ ) into $\mathfrak{U} \cdot($ the result sort of $\Sigma)+\cdot($ the characteristics of $\mathfrak{T}) \cdot \gamma$ is a many sorted function from $\mathfrak{U}^{\#}$. (the arity of $\Sigma$ ) into $\mathfrak{U} \cdot($ the result sort of $\Sigma$ ) by [19, (13)], [6, (12)], [17, (49)], [13, (11)]. $Q$ is T-extension by [35, (65)], [19, (72), (23)].

(18) Let us consider a non empty, non void signature $\Omega$, an $\Omega$-extension signature $\Sigma$, a non-empty algebra $\mathfrak{T}$ over $\Omega$, and a set $I$. Suppose $I \subseteq$ (the carrier of $\Sigma) \backslash$ (the carrier of $\Omega$ ). Let us consider a non-empty many sorted set $\mathcal{X}$ indexed by $I$. Then there exists a non-empty algebra $Q$ over $\Sigma$ such that

(i) $Q$ is $\mathfrak{T}$-extension, and

(ii) (the sorts of $Q)\lceil I=\mathcal{X}$.

The theorem is a consequence of (17) and (16).

Let $\Omega$ be a non empty, non void signature, $\Sigma$ be an $\Omega$-extension signature, and $\mathfrak{T}$ be an algebra over $\Omega$. One can verify that there exists an algebra over $\Sigma$ which is $\mathfrak{T}$-extension.

Let $\mathfrak{T}$ be a non-empty algebra over $\Omega$. One can check that there exists a non-empty algebra over $\Sigma$ which is $\mathfrak{T}$-extension.

Now we state the propositions:

(19) Let us consider a set $I$ and an object $a$. Then $\pi_{1}(I \otimes\{a\})$ is one-to-one.

(20) Let us consider signatures $\Sigma_{1}, \Sigma_{2}, E_{1}, E_{2}$. Suppose the many sorted signature of $\Sigma_{1}=$ the many sorted signature of $\Sigma_{2}$ and the many sorted signature of $E_{1}=$ the many sorted signature of $E_{2}$ and $E_{1}$ is an extension of $\Sigma_{1}$. Then $E_{2}$ is an extension of $\Sigma_{2}$.

Let $I$ be a set and $a$ be an object. Observe that $\pi_{1}(I \otimes\{a\})$ is one-to-one.

Let $a, b, c$ be non empty sets, $g$ be a function from $a$ into $b$, and $f$ be a function from $b$ into $c$. Note that the functor $f \cdot g$ yields a function from $a$ into $c$. Now we state the propositions:

(21) Let us consider a one-to-one function $f$. If $\mathcal{X}$ misses $Y$, then $f^{\circ} \mathcal{X}$ misses $f^{\circ} Y$.

(22) Let us consider a non empty, natural number $n$, a set $\mathcal{X}$, and a non empty signature $\Omega$. Then there exists a non empty, non void, PC correct w.r.t. $n, \mathrm{QC}$ correct QC language signature $Q$ over $\mathcal{X}$ such that 
(i) the carrier of $Q$ misses the carrier of $\Omega$, and

(ii) the carrier' of $Q$ misses the carrier' of $\Omega$.

Proof: Set $Q=$ the non empty, non void, PC correct w.r.t. $n$, QC correct $\mathrm{QC}$ language signature over $\mathcal{X}$. Reconsider $\alpha=$ (the carrier of $Q) \times\{$ the carrier of $\Omega\}$ as a non empty set. Reconsider $\beta=$ (the carrier' of $Q) \times\{$ the carrier' of $\Omega\}$ as a non empty set. Reconsider $f=\pi_{1}$ ( (the carrier of $Q) \otimes\{$ the carrier of $\Omega\}$ ) as a function from $\alpha$ into the carrier of $Q$. Reconsider $g=\pi_{1}(($ the carrier' of $Q) \otimes\{$ the carrier' of $\Omega\})$ as a function from $\beta$ into the carrier' of $Q$. Reconsider $f_{1}=f^{-1}$ as a function from the carrier of $Q$ into $\alpha$. Reconsider $g_{1}=g^{-1}$ as a function from the carrier' of $Q$ into $\beta$. Define $\mathcal{F}$ (object) $=f_{1} \cdot \$_{1}\left(\in(\text { the carrier of } Q)^{*}\right)$. Consider $f_{2}$ being a function such that $\operatorname{dom} f_{2}=(\text { the carrier of } Q)^{*}$ and for every object $p$ such that $p \in(\text { the carrier of } Q)^{*}$ holds $f_{2}(p)=\mathcal{F}(p)$ from [17. Sch. 3]. $\operatorname{rng} f_{2} \subseteq \alpha^{*}$. Reconsider $A_{6}=f_{2} \cdot($ the arity of $Q) \cdot g$ as a function from $\beta$ into $\alpha^{*}$. Reconsider $r_{3}=f_{1} \cdot($ the result sort of $Q) \cdot g$ as a function from $\beta$ into $\alpha$. Reconsider $f_{3}=f_{1}$ ((the formula sort of $Q)$ ) as an element of $\alpha$. Reconsider $c_{2}=g_{1}$. (the connectives of $Q$ ) as a finite sequence of elements of $\beta$. Reconsider $q_{5}=g_{1} \cdot$ (the quantifiers of $Q$ ) as a function from (the quantifier sort of $Q) \times \mathcal{X}$ into $\beta$. Set $Q_{3}=$ $\left\langle\left(\alpha\right.\right.$ qua non empty set), ( $\beta$ qua non empty set), $A_{6}, r_{3}, f_{3}, c_{2}$, (the quantifier sort of $Q$ qua set), $\left.q_{5}\right\rangle . Q_{3}$ is PC correct w.r.t. $n$ by [35, (27), (83)], [8, (12), (13)]. $Q_{3}$ is QC correct by [28, (52)], (21), [21, (87)], [18, (5)]. The carrier of $Q_{3}$ misses the carrier of $\Omega$. The carrier' of $Q_{3}$ misses the carrier' of $\Omega$.

Let $\Omega$ be a non empty signature. Let us note that there exists a non empty, non void signature which is $\Omega$-extension.

Let $\mathcal{X}$ be a set. Observe that there exists a non empty, non void QC language signature over $\mathcal{X}$ which is $\Omega$-extension.

Let $n$ be a non empty, natural number. Observe that there exists a non empty, non void, PC correct w.r.t. $n$, QC correct QC language signature over $\mathcal{X}$ which is $\Omega$-extension.

Let $\Omega$ be a non empty, non void signature, $\mathfrak{T}$ be a non-empty algebra over $\Omega, \mathcal{X}$ be a non empty yielding generator set of $\mathfrak{T}, \Sigma$ be an $\Omega$-extension, non empty, non void QC language signature over $\bigcup \mathcal{X}$, and $Y$ be an $\mathcal{X}$-tolerating many sorted set indexed by the carrier of $\Sigma$. We consider language structures over $\mathfrak{T}, Y$ and $\Sigma$ which extend free variable algebras over $\Sigma$ and algebras over $\Sigma, Y$ with substitution and are systems

〈sorts, a characteristics, free variables, a substitution operation, an equality 
where the sorts constitute a many sorted set indexed by the carrier of $\Sigma$, the characteristics is a many sorted function from (the sorts) ${ }^{\#}$ (the arity of $\Sigma$ ) into (the sorts) ( the result sort of $\Sigma$ ), the free variables constitute a double many sorted set of the sorts and the sorts, the substitution operation is a sort-preserving function from $U$ (the sorts) $\times \bigcup \llbracket Y$, the sorts $\rrbracket$ into $U$ (the sorts), the equality is a function from $\cup \llbracket$ the sorts of $\mathfrak{T}$, the sorts of $\mathfrak{T} \rrbracket$ into (the sorts)((the formula sort of $\Sigma)$ ).

Let $\Sigma$ be a non empty PC language signature and $L$ be an algebra over $\Sigma$. We say that $L$ is a language if and only if

(Def. 20) (the sorts of $L)(($ the formula sort of $\Sigma)$ ) is not empty.

One can check that every algebra over $\Sigma$ which is non-empty is also a language and there exists an algebra over $\Sigma$ which is a language.

Now we state the proposition:

(23) Let us consider a non void signature $\Omega$, an $\Omega$-extension, non void signature $\Sigma$, and algebras $A_{1}, A_{2}$ over $\Sigma$. Suppose the algebra of $A_{1}=$ the algebra of $A_{2}$. Then $A_{1}\left\lceil\Omega=A_{2} \mid \Omega\right.$.

Let $\Omega$ be a non empty, non void signature, $\mathfrak{T}$ be a non-empty algebra over $\Omega, \mathcal{X}$ be a non empty yielding generator set of $\mathfrak{T}$, and $\Sigma$ be an $\Omega$-extension, non empty, non void $\mathrm{QC}$ language signature over $\cup \mathcal{X}$. Observe that there exists a non empty yielding many sorted set indexed by the carrier of $\Sigma$ which is $\mathcal{X}$-tolerating.

Let $Y$ be an $\mathcal{X}$-tolerating, non empty yielding many sorted set indexed by the carrier of $\Sigma$. Let us observe that there exists a language structure over $\mathfrak{T}, Y$ and $\Sigma$ which is non-empty, language, and $\mathfrak{T}$-extension.

Let $L$ be a non-empty language structure over $\mathfrak{T}, Y$ and $\Sigma$. We say that $L$ is substitution correct (3) if and only if

(Def. 21) for every sort symbols $\sigma, \sigma_{1}$ of $\Sigma$ and for every element $\tau$ of $L$ from $\sigma$ and for every element $\tau_{1}$ of $L$ from $\sigma_{1}$ and for every element $y$ of $\bigcup Y$ such that $y \in Y(\sigma)$ holds $y \notin\left(\operatorname{vf} \tau_{1}\right)(\sigma)$ and if $y \notin\left(v f \tau_{1}\right)(\sigma)$, then $\tau_{1(y \leftarrow \tau)}=\tau_{1}$ and if $\tau_{1}=y \in Y\left(\sigma_{1}\right)$, then $\tau_{1(y \leftarrow \tau)}=\tau$ and for every element $x$ of $\cup Y$ such that $x \in Y(\sigma)$ holds $\tau_{1(x \leftarrow y)(y \leftarrow x)}=\tau_{1}$.

Let $\Omega$ be a non empty signature, $\Sigma$ be an $\Omega$-extension, non empty signature, $\mathcal{X}$ be a non empty yielding many sorted set indexed by the carrier of $\Omega$, and $Y$ be a set. Let us note that $\mathcal{X}$ extended by $(Y$, (the carrier of $\Sigma))$ is non empty yielding.

Let $\Omega$ be a non empty, non void signature, $\mathfrak{T}$ be a non-empty algebra over $\Omega, \mathcal{X}$ be a non empty yielding generator set of $\mathfrak{T}$, and $\Sigma$ be an $\Omega$-extension, non empty, non void $\mathrm{QC}$ language signature over $\cup \mathcal{X}$. Let us observe that there exists a language structure over $\mathfrak{T}, \mathcal{X}$ extended by $(\emptyset,($ the carrier of $\Sigma))$ and $\Sigma$ 
which is $(\mathcal{X}$ extended by $(\emptyset,($ the carrier of $\Sigma)))$-vf-yielding, non-empty, language, and $\mathfrak{T}$-extension.

Let $\mathcal{X}$ be a set, $\Sigma$ be a non empty QC language signature over $\mathcal{X}$, and $L$ be language algebra over $\Sigma$. Note that (the sorts of $L)(($ the formula sort of $\Sigma$ )) is non empty.

Let $\Omega$ be a non empty, non void signature, $\mathfrak{T}$ be a non-empty algebra over $\Omega, \mathcal{X}$ be a non empty yielding generator set of $\mathfrak{T}, \Sigma$ be an $\Omega$-extension, non empty, non void QC language signature over $\cup \mathcal{X}$, and $Y$ be an $\mathcal{X}$-tolerating, non empty yielding many sorted set indexed by the carrier of $\Sigma$.

A language over $Y$ and $\Sigma$ is language, $\mathfrak{T}$-extension language structure over $\mathfrak{T}, Y$ and $\Sigma$. Let $\Sigma$ be a non empty PC language signature and $L$ be language algebra over $\Sigma$.

A formula of $L$ is an element of (the sorts of $L)(($ the formula sort of $\Sigma)$ ). Let $n$ be a non empty, natural number and $\Sigma$ be a non void, non empty, PC correct w.r.t. $n \mathrm{PC}$ language signature. The functor true $L$ yielding a formula of $L$ is defined by the term

(Def. 22) (Den $(($ the connectives of $\Sigma)(n+5)(\in($ the carrier' of $\Sigma)), L))(\emptyset)$.

Let $\alpha$ be a formula of $L$. The functor $\neg \alpha$ yielding a formula of $L$ is defined by the term

(Def. 23) (Den $(($ the connectives of $\Sigma)(n)(\in($ the carrier' of $\Sigma)), L))(\langle\alpha\rangle)$.

Let $\beta$ be a formula of $L$. The functors: $\alpha \wedge \beta, \alpha \vee \beta, \alpha \Rightarrow \beta$, and $\alpha \Leftrightarrow \beta$ yielding formulae of $L$ are defined by terms,

(Def. 24) (Den $(($ the connectives of $\Sigma)(n+1)(\in($ the carrier' of $\Sigma)), L))(\langle\alpha, \beta\rangle)$,

(Def. 25) (Den $(($ the connectives of $\Sigma)(n+2)(\in($ the carrier' of $\Sigma)), L))(\langle\alpha, \beta\rangle)$,

(Def. 26) (Den $(($ the connectives of $\Sigma)(n+3)(\in($ the carrier' of $\Sigma)), L))(\langle\alpha, \beta\rangle)$,

(Def. 27) (Den $(($ the connectives of $\Sigma)(n+4)(\in($ the carrier' of $\Sigma)), L))(\langle\alpha, \beta\rangle)$, respectively. Let $\Omega$ be a non empty, non void signature and $\mathfrak{T}$ be a non-empty algebra over $\Omega$. Let us note that there exists a variable set in $\mathfrak{T}$ which is non empty.

Let $n$ be a non empty, natural number, $\mathcal{X}$ be a non empty yielding generator set of $\mathfrak{T}, \Sigma$ be an $\Omega$-extension, non void, non empty, PC correct w.r.t. $n$, QC correct QC language signature over $\bigcup \mathcal{X}, Y$ be an $\mathcal{X}$-tolerating, non empty yielding many sorted set indexed by the carrier of $\Sigma, L$ be a language over $Y$ and $\Sigma, \alpha$ be a formula of $L$, and $x$ be an element of $\cup \mathcal{X}$. The functors: $\forall x \alpha$ and $\exists_{x} \alpha$ yielding formulae of $L$ are defined by terms,

(Def. 28) (Den $(($ the quantifiers of $\Sigma)(1, x)(\in($ the carrier' of $\Sigma)), L))(\langle\alpha\rangle)$,

(Def. 29) (Den $(($ the quantifiers of $\Sigma)(2, x)(\in($ the carrier' of $\Sigma)), L))(\langle\alpha\rangle)$, 
respectively. Let $x, y$ be elements of $\bigcup \mathcal{X}$. The functors: $\forall_{x, y} \alpha$ and $\exists_{x, y} \alpha$ yielding formulae of $L$ are defined by terms,

(Def. 30) $\forall x(\forall y \alpha)$

(Def. 31) $\exists_{x}\left(\exists_{y} \alpha\right)$,

respectively. Let $x, y, z$ be elements of $\bigcup \mathcal{X}$. The functors: $\forall x, y, z \alpha$ and $\exists_{x, y, z} \alpha$ yielding formulae of $L$ are defined by terms,

(Def. 32) $\forall x, y\left(\forall_{z} \alpha\right)$,

(Def. 33) $\exists_{x, y}\left(\exists_{z} \alpha\right)$,

respectively. Let $\tau_{1}, \tau_{2}$ be objects. Given sort symbol $a$ of $\Omega$ such that $\tau_{1}, \tau_{2} \in$ (the sorts of $\mathfrak{T})(a)$. The functor $\tau_{1}={ }_{L} \tau_{2}$ yielding a formula of $L$ is defined by the term

(Def. 34) (the equality of $L)\left(\tau_{1}, \tau_{2}\right)$.

Let $L$ be a non-empty language over $Y$ and $\Sigma$. We say that $L$ is vf-QC-correct if and only if

(Def. 35) for every formulae $\alpha, \beta$ of $L$, vf $\neg \alpha=\operatorname{vf} \alpha$ and $\operatorname{vf}(\alpha \wedge \beta)=\operatorname{vf} \alpha \cup \operatorname{vf} \beta$ and $\operatorname{vf}(\alpha \vee \beta)=\operatorname{vf} \alpha \cup \operatorname{vf} \beta$ and $\operatorname{vf}(\alpha \Rightarrow \beta)=\operatorname{vf} \alpha \cup \operatorname{vf} \beta$ and $\operatorname{vf}(\alpha \Leftrightarrow$ $\beta)=\operatorname{vf} \alpha \cup \operatorname{vf} \beta$ and $\operatorname{vf}$ true $_{L}=\mathbf{0}$. (the carrier of $\Sigma$ ) and for every element $x$ of $\bigcup \mathcal{X}$ and for every sort symbol $a$ of $\Sigma$ such that $x \in \mathcal{X}(a)$ holds vf $\forall_{x} \alpha=\operatorname{vf} \alpha \backslash a$-singleton $x$ and vf $\exists_{x} \alpha=$ vf $\alpha \backslash a$-singleton $x$.

Let $L$ be a non-empty, T-extension language over $Y$ and $\Sigma$. We say that $L$ is vf finite if and only if

(Def. 36) for every sort symbol $\sigma$ of $\Sigma$ and for every element $\tau$ of $L$ from $\sigma, \operatorname{vf} \tau$ is finite-yielding.

We say that $L$ is substitution for $\forall$ and $\exists$ if and only if

(Def. 37) for every formula $\alpha$ of $L$ and for every element $x$ of $\cup \mathcal{X}$ and for every sort symbols $\sigma, \sigma_{1}$ of $\Sigma$ and for every element $\tau$ of $L$ from $\sigma$ such that $x \in \mathcal{X}\left(\sigma_{1}\right)$ for every element $y$ of $\bigcup Y$ such that $y \in Y(\sigma)$ holds if $x=$ $y$, then $\left(\forall_{x} \alpha\right)_{(y \leftarrow \tau)}=\forall x \alpha$ and $\left(\exists_{x} \alpha\right)_{(y \leftarrow \tau)}=\exists_{x} \alpha$ and if $x \neq y$ and $x \in(\operatorname{vf} \tau)\left(\sigma_{1}\right)$, then there exists an element $z$ of $\cup \mathcal{X}$ and there exist elements $x_{0}, z_{0}$ of $\bigcup Y$ such that $x=x_{0}$ and $z_{0}=z=$ the element of $\left(\mathcal{X}\left(\sigma_{1}\right) \backslash(\operatorname{vf} \tau)\left(\sigma_{1}\right)\right) \backslash(\operatorname{vf} \alpha)\left(\sigma_{1}\right)$ and $\left(\forall_{x} \alpha\right)_{(y \leftarrow \tau)}=\forall_{z}\left(\alpha_{\left(x_{0} \leftarrow z_{0}\right)(y \leftarrow \tau)}\right)$ and $\left(\exists_{x} \alpha\right)_{(y \leftarrow \tau)}=\exists_{z}\left(\alpha_{\left(x_{0} \leftarrow z_{0}\right)(y \leftarrow \tau)}\right)$ and if $x \neq y$ and $x \notin(\operatorname{vf} \tau)(\sigma)$, then $\left(\forall_{x} \alpha\right)_{(y \leftarrow \tau)}=\forall_{x}\left(\alpha_{(y \leftarrow \tau)}\right)$ and $\left(\exists_{x} \alpha\right)_{(y \leftarrow \tau)}=\exists_{x}\left(\alpha_{(y \leftarrow \tau)}\right)$.

Now we state the propositions:

(24) Let us consider a non void signature $\Omega$, an algebra $\mathfrak{T}$ over $\Omega$, a many sorted subset $\mathcal{X}$ of the sorts of $\mathfrak{T}$, an $\Omega$-extension, non void signature $\Sigma$, and a $\mathfrak{T}$-extension algebra $Q$ over $\Sigma$. Then $\mathcal{X}$ extended by $(\emptyset$, (the carrier of $\Sigma))$ 
is a many sorted subset of the sorts of $Q$. The theorem is a consequence of $(16)$.

(25) Let us consider a non void signature $\Omega$, an algebra $\mathfrak{T}$ over $\Omega$, a many sorted subset $\mathcal{X}$ of the sorts of $\mathfrak{T}$, and an $\Omega$-extension, non void signature $\Sigma$. Then $\bigcup(\mathcal{X}$ extended by $(\emptyset,($ the carrier of $\Sigma)))=\bigcup \mathcal{X}$.

(26) Let us consider a non empty, natural number $n$, a non empty set $\mathcal{X}$, a non empty, non void, PC correct w.r.t. $n$, QC correct QC language signature $\Sigma$ over $\mathcal{X}$, and language algebra $Q$ over $\Sigma$. Then

(i) $\emptyset \in \operatorname{Args}(($ the connectives of $\Sigma)(n+5)(\in($ the carrier' of $\Sigma)), Q)$, and

(ii) for every formula $\alpha$ of $Q,\langle\alpha\rangle \in \operatorname{Args}(($ the connectives of $\Sigma)(n)(\in$ (the carrier' of $\Sigma)$ ), $Q$ ) and for every formula $\beta$ of $Q,\langle\alpha, \beta\rangle \in \operatorname{Args}(($ the connectives of $\Sigma)(n+1)(\in($ the carrier' of $\Sigma)), Q)$ and $\ldots$ and $\langle\alpha$, $\beta\rangle \in \operatorname{Args}(($ the connectives of $\Sigma)(n+4)(\in($ the carrier' of $\Sigma)), Q)$ and for every element $x$ of $\mathcal{X},\langle\alpha\rangle \in \operatorname{Args}(($ the quantifiers of $\Sigma)(1, x)(\in$ (the carrier' of $\Sigma)), Q)$ and $\langle\alpha\rangle \in \operatorname{Args}(($ the quantifiers of $\Sigma)(2, x)(\in$ (the carrier' of $\Sigma)$ ), $Q$ ).

The theorem is a consequence of (4), (5), and (6).

(27) Let us consider a non empty, natural number $n$, a non empty, non void signature $\Omega$, a non-empty algebra $\mathfrak{T}$ over $\Omega$, a non empty yielding generator set $\mathcal{X}$ of $\mathfrak{T}$, an $\Omega$-extension, non void, non empty, PC correct w.r.t. $n$, QC correct QC language signature $\Sigma$ over $\cup \mathcal{X}$, an $\mathcal{X}$-tolerating, non empty yielding many sorted set $Y$ indexed by the carrier of $\Sigma$, a non-empty language $L$ over $Y$ and $\Sigma$, an element $x$ of $\bigcup Y$, an element $\tau$ of $\bigcup$ (the sorts of $L$ ), and a sort symbol $\sigma$ of $\Sigma$. Suppose $x \in Y(\sigma)$ and $\tau \in$ (the sorts of $L)(\sigma)$. Then

(i) for every element $a$ of $\operatorname{Args}(($ the connectives of $\Sigma)(n+5)(\in$ (the carrier' of $\Sigma)), L)$ such that $a=\emptyset$ holds $a_{(x \leftarrow \tau)}=\emptyset$, and

(ii) for every formula $\alpha$ of $L$, for every element $a$ of $\operatorname{Args}(($ the connectives of $\Sigma)(n)(\in($ the carrier' of $\Sigma)), L)$ such that $\langle\alpha\rangle=a$ holds $a_{(x \leftarrow \tau)}=$ $\left\langle\alpha_{(x \leftarrow \tau)}\right\rangle$ and for every formula $\beta$ of $L$, for every element $a$ of $\operatorname{Args}(($ the connectives of $\Sigma)(n+1)(\in($ the carrier' of $\Sigma)), L)$ such that $\langle\alpha, \beta\rangle=a$ holds $a_{(x \leftarrow \tau)}=\left\langle\alpha_{(x \leftarrow \tau)}, \beta_{(x \leftarrow \tau)}\right\rangle$ and ... and for every element $a$ of $\operatorname{Args}(($ the connectives of $\Sigma)(n+4)(\in($ the carrier' of $\Sigma)), L)$ such that $\langle\alpha, \beta\rangle=a$ holds $a_{(x \leftarrow \tau)}=\left\langle\alpha_{(x \leftarrow \tau)}, \beta_{(x \leftarrow \tau)}\right\rangle$ and for every element $z$ of $\bigcup \mathcal{X}$, for every element $a$ of $\operatorname{Args}(($ the quantifiers of $\Sigma)(1, z)(\in$ (the carrier' of $\Sigma)$ ), $L)$ such that $\langle\alpha\rangle=a$ holds $a_{(x \leftarrow \tau)}=\left\langle\alpha_{(x \leftarrow \tau)}\right\rangle$ and for every element $a$ of $\operatorname{Args}(($ the quantifiers of $\Sigma)(2, z)(\in$ (the carrier' of $\Sigma)$ ), $L)$ such that $\langle\alpha\rangle=a$ holds $a_{(x \leftarrow \tau)}=\left\langle\alpha_{(x \leftarrow \tau)}\right\rangle$. 
ProOF: for every element $a$ of $\operatorname{Args}(($ the connectives of $\Sigma)(n+1)(\in$ (the carrier' of $\Sigma)), L)$ such that $\langle\alpha, \beta\rangle=a$ holds $a_{(x \leftarrow \tau)}=\left\langle\alpha_{(x \leftarrow \tau)}, \beta_{(x \leftarrow \tau)}\right\rangle$ and $\ldots$ and for every element $a$ of $\operatorname{Args}(($ the connectives of $\Sigma)(n+4)(\epsilon$ (the carrier' of $\Sigma)$ ), $L)$ such that $\langle\alpha, \beta\rangle=a$ holds $a_{(x \leftarrow \tau)}=\left\langle\alpha_{(x \leftarrow \tau)}\right.$, $\left.\beta_{(x \leftarrow \tau)}\right\rangle$ by [7, (2)], [12, (44), (89), (1)]. Set $o=$ (the quantifiers of $\Sigma)(2, z)(\in($ the carrier' of $\Sigma))$. Consider $j$ being a sort symbol of $\Sigma$ such that $j=\operatorname{Arity}(o)(1)$ and there exists an element $\alpha$ of $L$ from $j$ such that $\alpha=a(1)$ and $a_{(x \leftarrow \tau)}(1)=\alpha_{(x \leftarrow \tau)}$.

(28) Let us consider a non empty, natural number $n$, a non empty, non void signature $\Omega$, a non-empty algebra $\mathfrak{T}$ over $\Omega$, a non empty yielding generator set $\mathcal{X}$ of $\mathfrak{T}$, an $\Omega$-extension, non empty, non void, PC correct w.r.t. $n$, QC correct $\mathrm{QC}$ language signature $\Sigma$ over $\bigcup \mathcal{X}$, an $\mathcal{X}$-tolerating, non empty yielding many sorted set $Y$ indexed by the carrier of $\Sigma$, and a non-empty language $L$ over $Y$ and $\Sigma$. Suppose $L$ is substitution correct (1) and $Y$ is a many sorted subset of the sorts of $L$. Let us consider elements $x, y$ of $\cup Y$ and a sort symbol $a$ of $\Sigma$. Suppose $x, y \in Y(a)$. Let us consider a formula $\alpha$ of $L$. Then

(i) $(\neg \alpha)_{(x \leftarrow y)}=\neg\left(\alpha_{(x \leftarrow y)}\right)$, and

(ii) for every formula $\beta$ of $L,(\alpha \wedge \beta)_{(x \leftarrow y)}=\alpha_{(x \leftarrow y)} \wedge \beta_{(x \leftarrow y)}$ and $(\alpha \vee$ $\beta)_{(x \leftarrow y)}=\alpha_{(x \leftarrow y)} \vee \beta_{(x \leftarrow y)}$ and $(\alpha \Rightarrow \beta)_{(x \leftarrow y)}=\alpha_{(x \leftarrow y)} \Rightarrow \beta_{(x \leftarrow y)}$ and $(\alpha \Leftrightarrow \beta)_{(x \leftarrow y)}=\alpha_{(x \leftarrow y)} \Leftrightarrow \beta_{(x \leftarrow y)}$ and $\left(\operatorname{true}_{L}\right)_{(x \leftarrow y)}=\operatorname{true}_{L}$.

The theorem is a consequence of (26), (27), and (14).

\section{Algorithmic Theory}

Let $\Omega$ be a non empty, non void signature, $\mathfrak{T}$ be a non-empty algebra over $\Omega$, $\mathcal{X}$ be a non empty yielding generator set of $\mathfrak{T}, \Sigma$ be an $\Omega$-extension, non void, non empty $\mathrm{QC}$ language signature over $\bigcup \mathcal{X}$, and $Y$ be an $\mathcal{X}$-tolerating many sorted set indexed by the carrier of $\Sigma$. We consider bialgebra structures over $\Sigma, Y$ which extend language structures over $\mathfrak{T}, Y$ and $\Sigma$ and program algebra structures of $\Omega, \mathfrak{T}$, and $\mathcal{X}$ and are systems

〈sorts, a characteristics, free variables, a substitution operation,

an equality, a carrier, a characteristic, assignments $\rangle$

where the sorts constitute a many sorted set indexed by the carrier of $\Sigma$, the characteristics is a many sorted function from (the sorts) ${ }^{\#}$ (the arity of $\Sigma$ ) into (the sorts) ( the result sort of $\Sigma$ ), the free variables constitute a double many sorted set of the sorts and the sorts, the substitution operation is a sort-preserving 
function from $U($ the sorts $) \times \bigcup \llbracket Y$, the sorts $\rrbracket$ into $U($ the sorts $)$, the equality is a function from $\bigcup \llbracket$ the sorts of $\mathfrak{T}$, the sorts of $\mathfrak{T} \rrbracket$ into (the sorts)((the formula sort of $\Sigma)$ ), the carrier is a set, the characteristic is a finite sequence of operational functions of the carrier, the assignments constitute a function from $\bigcup \llbracket \mathcal{X}$, the sorts of $\mathfrak{T} \rrbracket$ into the carrier.

Let us note that there exists a non void, non empty algorithmic language signature of $\bigcup \mathcal{X}$ which is $\Omega$-extension.

Let $\Sigma$ be an $\Omega$-extension, non void, non empty algorithmic language signature of $\bigcup \mathcal{X}, Y$ be an $\mathcal{X}$-tolerating many sorted set indexed by the carrier of $\Sigma$, and $L$ be a bialgebra structure over $\Sigma, Y$. We say that $L$ is AL correct if and only if

(Def. 38) the carrier of $L=($ the sorts of $L)(($ the program sort of $\Sigma))$.

Let $\Sigma$ be a 1 -sorted structure. We introduce $\Sigma$ is 1 s-empty as a synonym of $\Sigma$ is empty.

Let $\Sigma$ be a universal algebra structure. We introduce $\Sigma$ is ua-non-empty as a synonym of $\Sigma$ is non-empty.

Let $\Omega$ be a non empty, non void signature, $\mathfrak{T}$ be a non-empty algebra over $\Omega$, $\mathcal{X}$ be a non empty yielding generator set of $\mathfrak{T}, \Sigma$ be an $\Omega$-extension, non void, non empty algorithmic language signature of $\bigcup \mathcal{X}$, and $Y$ be an $\mathcal{X}$-tolerating many sorted set indexed by the carrier of $\Sigma$. Let us observe that there exists a strict bialgebra structure over $\Sigma, Y$ which is non 1s-empty.

Let $n$ be a non empty natural number and $\Sigma$ be an essential, $\Omega$-extension, PC correct w.r.t. $n$, non void, non empty, feasible algorithmic language signature of $\cup \mathcal{X}$. One can check that there exists a non 1s-empty, strict bialgebra structure over $\Sigma, Y$ which is non-empty, language, AL correct, quasi total, partial, ua-nonempty, and $\mathfrak{T}$-extension and has empty-instruction, catenation, if-instruction, and while-instruction.

Let us consider pre-if-while algebras $\mathfrak{U}_{1}, \mathfrak{U}_{2}$.

Let us assume that the universal algebra structure of $\mathfrak{U}_{1}=$ the universal algebra structure of $\mathfrak{U}_{2}$. Now we state the propositions:

(i) EmptyIns $_{\mathfrak{U}_{1}}=$ EmptyIns $_{\mathfrak{U}_{2}}$, and

(ii) for every elements $I_{1}, J_{1}$ of $\mathfrak{U}_{1}$ and for every elements $I_{2}, J_{2}$ of $\mathfrak{U}_{2}$ such that $I_{1}=I_{2}$ and $J_{1}=J_{2}$ holds $I_{1} ; J_{1}=I_{2} ; J_{2}$ and while $I_{1}$ do $J_{1}=$ while $I_{2}$ do $J_{2}$ and for every element $C_{1}$ of $\mathfrak{U}_{1}$ and for every element $C_{2}$ of $\mathfrak{U}_{2}$ such that $C_{1}=C_{2}$ holds if $C_{1}$ then $I_{1}$ else $J_{1}=$ if $C_{2}$ then $I_{2}$ else $J_{2}$.

(30) ElementaryInstructions $\mathfrak{U}_{1}=$ ElementaryInstructions $_{\mathfrak{U}_{2}}$.

Now we state the propositions:

(31) Let us consider universal algebras $\mathfrak{U}_{1}, \mathfrak{U}_{2}$. Suppose the universal algebra structure of $\mathfrak{U}_{1}=$ the universal algebra structure of $\mathfrak{U}_{2}$. Let us consider a 
subset $\Sigma_{1}$ of $\mathfrak{U}_{1}$ and a subset $\Sigma_{2}$ of $\mathfrak{U}_{2}$. Suppose $\Sigma_{1}=\Sigma_{2}$. Let us consider an operation $o_{1}$ of $\mathfrak{U}_{1}$ and an operation $o_{2}$ of $\mathfrak{U}_{2}$. Suppose $o_{1}=o_{2}$. If $\Sigma_{1}$ is closed on $o_{1}$, then $\Sigma_{2}$ is closed on $o_{2}$.

(32) Let us consider universal algebras $\mathfrak{U}_{1}, \mathfrak{U}_{2}$. Suppose the universal algebra structure of $\mathfrak{U}_{1}=$ the universal algebra structure of $\mathfrak{U}_{2}$. Let us consider a subset $\Sigma_{1}$ of $\mathfrak{U}_{1}$ and a subset $\Sigma_{2}$ of $\mathfrak{U}_{2}$. Suppose $\Sigma_{1}=\Sigma_{2}$. If $\Sigma_{1}$ is operations closed, then $\Sigma_{2}$ is operations closed.

Let us consider universal algebras $\mathfrak{U}_{1}, \mathfrak{U}_{2}$.

Let us assume that the universal algebra structure of $\mathfrak{U}_{1}=$ the universal algebra structure of $\mathfrak{U}_{2}$. Now we state the propositions:

(33) Every generator set of $\mathfrak{U}_{1}$ is a generator set of $\mathfrak{U}_{2}$. The theorem is a consequence of (32).

(34) signature $\mathfrak{U}_{1}=$ signature $\mathfrak{U}_{2}$.

Let $n$ be a non empty, natural number, $\Omega$ be a non empty, non void signature, $\mathfrak{T}$ be a non-empty algebra over $\Omega, \mathcal{X}$ be a non empty yielding generator set of $\mathfrak{T}$, and $\Sigma$ be an essential, $\Omega$-extension, non void, non empty, PC correct w.r.t. $n$, QC correct algorithmic language signature of $\bigcup \mathcal{X}$. Let us observe that there exists a non-empty, quasi total, partial, ua-non-empty, a language, non 1sempty, $\mathfrak{T}$-extension bialgebra structure over $\Sigma, \mathcal{X}$ extended by $(\emptyset$, (the carrier of $\Sigma$ )) with empty-instruction, catenation, if-instruction, and while-instruction which is AL correct, vf-QC-correct, vf-correct, vf finite, substitution correct (1), substitution for $\forall$ and $\exists$, non degenerated, well founded, and E.C.I.W.-strict.

Let $\Sigma$ be an essential, $\Omega$-extension, non empty, non void, PC correct w.r.t. $n, \mathrm{QC}$ correct, $\mathrm{AL}$ correct w.r.t. $n$ algorithmic language signature of $\bigcup \mathcal{X}$.

An if-while algebra of $\mathcal{X}$ over $\Sigma$ is an AL correct, vf-QC-correct, vf-correct, substitution correct (1), substitution for $\forall$ and $\exists$, non degenerated, well founded, E.C.I.W.-strict, non-empty, quasi total, partial, ua-non-empty, a language, non 1s-empty, T-extension bialgebra structure over $\Sigma, \mathcal{X}$ extended by $(\emptyset$, (the carrier of $\Sigma$ )) with empty-instruction, catenation, if-instruction, and while-instruction. Let $n$ be a non empty natural number, $L$ be an if-while algebra of $\mathcal{X}$ over $\Sigma, K$ be a formula of $L$, and $P$ be an algorithm of $L$. The functors: $P \cdot K$, $\uplus(P, K)$, and $\cap(P, K)$ yielding formulae of $L$ are defined by terms,

(Def. 39) (Den $(($ the connectives of $\Sigma)(n+6)(\in($ the carrier' of $\Sigma)), L))(\langle P, K\rangle)$,

(Def. 40) (Den $(($ the connectives of $\Sigma)(n+7)(\in($ the carrier' of $\Sigma)), L))(\langle P, K\rangle)$,

(Def. 41) (Den $(($ the connectives of $\Sigma)(n+8)(\in($ the carrier' of $\Sigma)), L))(\langle P, K\rangle)$, respectively. Let $\Sigma$ be a non empty, non void, PC correct w.r.t. $n$ PC language signature, $L$ be language algebra over $\Sigma$, and $F$ be a subset of (the sorts of $L)(($ the formula sort of $\Sigma)$ ). We say that $F$ is PC-closed if and only if 
(Def. 42) for every formulae $\alpha, \beta, \gamma$ of $L, \alpha \Rightarrow(\beta \Rightarrow \alpha),(\alpha \Rightarrow(\beta \Rightarrow \gamma)) \Rightarrow((\alpha \Rightarrow$ $\beta) \Rightarrow(\alpha \Rightarrow \gamma)),(\neg \alpha \Rightarrow \neg \beta) \Rightarrow(\beta \Rightarrow \alpha), \alpha \Rightarrow(\alpha \vee \beta), \alpha \Rightarrow(\beta \vee \alpha)$, $(\alpha \Rightarrow \gamma) \Rightarrow((\beta \Rightarrow \gamma) \Rightarrow((\alpha \vee \beta) \Rightarrow \gamma)),(\alpha \wedge \beta) \Rightarrow \alpha,(\alpha \wedge \beta) \Rightarrow \beta$, $\alpha \Rightarrow(\beta \Rightarrow(\alpha \wedge \beta)),(\alpha \wedge \neg \alpha) \Rightarrow \beta,(\alpha \Rightarrow \beta) \Rightarrow((\alpha \Rightarrow \neg \beta) \Rightarrow \neg \alpha), \alpha \vee \neg \alpha$, $(\alpha \Leftrightarrow \beta) \Rightarrow(\alpha \Rightarrow \beta),(\alpha \Leftrightarrow \beta) \Rightarrow(\beta \Rightarrow \alpha),((\alpha \Rightarrow \beta) \wedge(\beta \Rightarrow \alpha)) \Rightarrow(\alpha \Leftrightarrow$ $\beta), \operatorname{true}_{L},\left(\operatorname{true}_{L} \wedge \alpha\right) \Leftrightarrow \alpha,\left(\operatorname{true}_{L} \vee \alpha\right) \Leftrightarrow \operatorname{true}_{L} \in F$ and if $\alpha, \alpha \Rightarrow \beta \in F$, then $\beta \in F$.

Let $\Omega$ be a non empty, non void signature, $\mathfrak{T}$ be a non-empty algebra over $\Omega$, $\mathcal{X}$ be a non empty yielding generator set of $\mathfrak{T}, \Sigma$ be an $\Omega$-extension, non empty, non void, $\mathrm{PC}$ correct w.r.t. $n$, QC correct QC language signature over $\bigcup \mathcal{X}$, and $L$ be a non-empty language over $\mathcal{X}$ extended by $(\emptyset$, (the carrier of $\Sigma$ )) and $\Sigma$. We say that $F$ is QC closed if and only if

(Def. 43) for every elements $\alpha, \beta$ of (the sorts of $L)(($ the formula sort of $\Sigma)$ ) and for every element $x$ of $\bigcup \mathcal{X}$, for every sort symbol $a$ of $\Omega$, for every element $\tau$ of $\bigcup($ the sorts of $L)$ such that $x \in(\mathcal{X}$ extended by $(\emptyset,($ the carrier of $\Sigma)))(a)$ and $\tau \in($ the sorts of $L)(a)$ for every element $y$ of $\bigcup(\mathcal{X}$ extended by $(\emptyset$, (the carrier of $\Sigma)$ )) such that $x=y$ holds $\forall_{x} \alpha \Rightarrow \alpha_{(y \leftarrow \tau)} \in F$ and if $x \in \mathcal{X}(a)$ and $x \notin(\operatorname{vf} \alpha)(a)$, then $\forall x(\alpha \Rightarrow \beta) \Rightarrow(\alpha \Rightarrow \forall x \beta) \in F$ and $\neg \exists_{x} \alpha \Leftrightarrow$ $\forall x(\neg \alpha), \exists_{x}(\neg \alpha) \Leftrightarrow \neg \forall x \alpha \in F$ and if $\alpha \in F$, then $\forall x \alpha \in F$.

Let $L$ be a non-empty, $\mathfrak{T}$-extension language over $\mathcal{X}$ extended by $(\emptyset$, (the carrier of $\Sigma)$ ) and $\Sigma$. We say that $L$ is substitution eq-correct if and only if

(Def. 44) for every element $x_{0}$ of $\bigcup(\mathcal{X}$ extended by $(\emptyset$, (the carrier of $\left.\Sigma))\right)$ and for every sort symbols $\sigma, \sigma_{1}$ of $\Sigma$ such that $x_{0} \in \mathcal{X}(\sigma)$ for every element $\tau$ of $L$ from $\sigma$ for every elements $\tau_{1}, \tau_{2}$ of $L$ from $\sigma_{1},\left(\tau_{1}={ }_{L} \tau_{2}\right)_{\left(x_{0} \leftarrow \tau\right)}=$ $\tau_{1\left(x_{0} \leftarrow \tau\right)}={ }_{L} \tau_{2\left(x_{0} \leftarrow \tau\right)}$.

We say that $L$ is vf-eq-correct if and only if

(Def. 45) for every sort symbol $\sigma$ of $\Sigma$, for every elements $\tau_{1}$, $\tau_{2}$ of $L$ from $\sigma$, $\operatorname{vf}\left(\tau_{1}={ }_{L} \tau_{2}\right)=\operatorname{vf} \tau_{1} \cup \operatorname{vf} \tau_{2}$ and for every sort symbol $\sigma$ of $\Sigma$ and for every element $\tau$ of $L$ from $\sigma$ such that $\tau \in \mathcal{X}(\sigma)$ holds vf $\tau=\sigma$-singleton $\tau$.

We say that $F$ has equality if and only if

(Def. 46) for every element $\tau$ of $\mathfrak{T}, \tau={ }_{L} \tau \in F$ and for every sort symbol $b$ of $\Sigma$ and for every elements $\tau_{1}, \tau_{2}$ of $L$ from $b$ and for every element $x$ of $\bigcup(\mathcal{X}$ extended by $(\emptyset,($ the carrier of $\Sigma)))$ such that $x \in \mathcal{X}(b)$ holds for every sort symbol $c$ of $\Sigma$ such that $c \in$ the carrier of $\Omega$ for every element $\tau$ of $L$ from $c,\left(\tau_{1}={ }_{L} \tau_{2}\right) \Rightarrow\left(\tau_{\left(x \leftarrow \tau_{1}\right)}={ }_{L} \tau_{\left(x \leftarrow \tau_{2}\right)}\right) \in F$ and for every formula $\alpha$ of $L$, $\left(\tau_{1}={ }_{L} \tau_{2}\right) \Rightarrow\left(\alpha_{\left(x \leftarrow \tau_{1}\right)} \Rightarrow \alpha_{\left(x \leftarrow \tau_{2}\right)}\right) \in F$.

Let $n$ be a non empty, natural number, $\mathfrak{T}$ be a non-empty free variable algebra over $\Omega, \mathcal{X}$ be a non-empty generator set of $\mathfrak{T}, \Sigma$ be an essential, $\Omega$ extension, non empty, non void, PC correct w.r.t. $n$, QC correct, $\mathrm{AL}$ correct 
w.r.t. $n$ algorithmic language signature of $\bigcup \mathcal{X}, L$ be a non 1s-empty if-while algebra of $\mathcal{X}$ over $\Sigma$, and $\varphi$ be a formula of $L$. We say that $F$ is AL closed w.r.t $\varphi$ if and only if

(Def. 47) for every formulae $\alpha, \beta$ of $L$, for every algorithm $M$ of $L, M \cdot(\alpha \wedge \beta) \Leftrightarrow$ $(M \cdot \alpha \wedge(M \cdot \beta)), M \cdot(\alpha \vee \beta) \Leftrightarrow(M \cdot \alpha \vee(M \cdot \beta)), \mathbb{U}(M, \alpha) \Leftrightarrow(\alpha \vee$ $\mathbb{U}(M, M \cdot \alpha)), \cap(M, \alpha) \Leftrightarrow(\alpha \wedge \cap(M, M \cdot \alpha)) \in F$ and if $\alpha \Rightarrow \beta \in F$, then $\mathbb{U}(M, \alpha) \Rightarrow \mathbb{U}(M, \beta), \cap(M, \alpha) \Rightarrow \cap(M, \beta) \in F$ and for every sort symbol $a$ of $\Omega$ and for every element $x$ of $\mathcal{X}(a)$ and for every element $x_{0}$ of $\bigcup(\mathcal{X}$ extended by $(\emptyset,($ the carrier of $\Sigma)))$ such that $x=x_{0}$ for every element $\tau$ of (the sorts of $\mathfrak{T})(a)$ for every element $\tau_{1}$ of $\bigcup$ (the sorts of $L$ ) such that $\tau_{1}=\tau$ holds $x:{ }_{L} \tau \cdot \alpha \Leftrightarrow \alpha_{\left(x_{0} \leftarrow \tau_{1}\right)} \in F$ and for every element $y$ of $\mathcal{X}(a)$ such that $y \notin(\operatorname{vf} \tau)(a)$ for every element $y_{0}$ of $\bigcup(\mathcal{X}$ extended by $(\emptyset$, (the carrier of $\Sigma))$ ) such that $y=y_{0}$ holds $x:={ }_{L} \tau \cdot \exists_{x} \alpha \Leftrightarrow \exists_{y}\left(x:={ }_{L} \tau \cdot\left(y:{ }_{L}\left({ }^{\circledR} x\right)\right.\right.$. $\left.\left.\alpha_{\left(x_{0} \leftarrow y_{0}\right)}\right)\right) \in F$ and $x:={ }_{L} \tau \cdot \alpha \Rightarrow \exists_{x} \alpha \in F$ and for every algorithms $M$, $M_{1}, M_{2}$ of $L,\left(M ; M_{1}\right) \cdot \alpha \Leftrightarrow\left(M \cdot\left(M_{1} \cdot \alpha\right)\right)$, if $M$ then $M_{1}$ else $M_{2} \cdot \alpha \Leftrightarrow$ $\left(\left(M \cdot \varphi \wedge\left(M \cdot\left(M_{1} \cdot \alpha\right)\right)\right) \vee\left(M \cdot \neg \varphi \wedge\left(M \cdot\left(M_{2} \cdot \alpha\right)\right)\right)\right)$, while $M$ do $M_{1} \cdot \alpha \Leftrightarrow$ $\left((M \cdot \neg \varphi \wedge \alpha) \vee\left(M \cdot \varphi \wedge\left(M \cdot\left(M_{1} \cdot\left(\right.\right.\right.\right.\right.$ while $M$ do $\left.\left.\left.\left.\left.M_{1} \cdot \alpha\right)\right)\right)\right)\right) \in F$.

Let $n$ be a non empty natural number, $\Sigma$ be a non empty, non void, PC correct w.r.t. $n$ PC language signature, and $L$ be language algebra over $\Sigma$. One can check that $\Omega_{\text {(the sorts of } L)((\text { the formula sort of } \Sigma) \text { ) }}$ is PC-closed and every subset of (the sorts of $L)$ ((the formula sort of $\Sigma)$ ) which is PC-closed is also non empty and there exists a subset of (the sorts of $L)(($ the formula sort of $\Sigma)$ ) which is PC-closed.

Let $\Omega$ be a non empty, non void signature, $\mathfrak{T}$ be a non-empty algebra over $\Omega, \mathcal{X}$ be a non empty yielding generator set of $\mathfrak{T}, \Sigma$ be an $\Omega$-extension, non empty, non void, PC correct w.r.t. $n$, QC correct QC language signature over $\cup \mathcal{X}$, and $L$ be a non-empty language over $\mathcal{X}$ extended by $(\emptyset$, (the carrier of $\Sigma)$ )

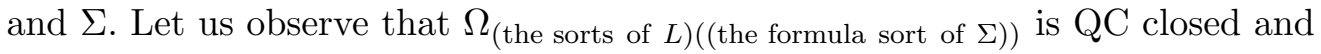
there exists a subset of (the sorts of $L)(($ the formula sort of $\Sigma)$ ) which is QC closed and PC-closed.

Let $L$ be a non-empty, $\mathfrak{T}$-extension language over $\mathcal{X}$ extended by $(\emptyset$, (the carrier

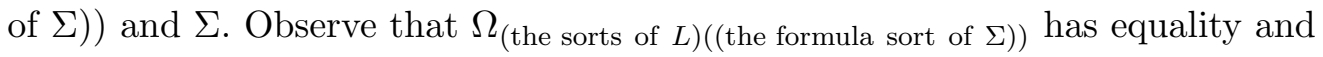
there exists a subset of (the sorts of $L)(($ the formula sort of $\Sigma)$ ) which is QC closed and PC-closed and has equality.

Let $\Sigma$ be a non empty, non void, PC correct w.r.t. $n$ PC language signature and $L$ be language algebra over $\Sigma$.

A PC theory of $L$ is a PC-closed subset of (the sorts of $L)(($ the formula sort of $\Sigma)$ ). Let $\Omega$ be a non empty, non void signature, $\mathfrak{T}$ be a non-empty algebra over $\Omega, \mathcal{X}$ be a non empty yielding generator set of $\mathfrak{T}, \Sigma$ be an $\Omega$-extension, non empty, non void, $\mathrm{PC}$ correct w.r.t. $n$, QC correct $\mathrm{QC}$ language signature over 
$\bigcup \mathcal{X}$, and $L$ be a non-empty language over $\mathcal{X}$ extended by $(\emptyset,($ the carrier of $\Sigma))$ and $\Sigma$.

A QC theory of $L$ is a QC closed, PC-closed subset of (the sorts of $L)(($ the formula sort of $\Sigma)$ ). Let $L$ be a non-empty, $\mathfrak{T}$-extension language over $\mathcal{X}$ extended by $(\emptyset$, (the carrier of $\Sigma))$ and $\Sigma$.

A QC theory of $L$ with equality is a QC closed, PC-closed subset of (the sorts of $L)(($ the formula sort of $\Sigma)$ ) with equality. Let $n$ be a non empty, natural number, $\mathfrak{T}$ be a non-empty free variable algebra over $\Omega, \mathcal{X}$ be a non-empty generator set of $\mathfrak{T}, \Sigma$ be an essential, $\Omega$-extension, non empty, non void, PC correct w.r.t. $n$, QC correct, $\mathrm{AL}$ correct w.r.t. $n$ algorithmic language signature of $\bigcup \mathcal{X}, L$ be a non 1s-empty if-while algebra of $\mathcal{X}$ over $\Sigma$, and $\varphi$ be a formula of $L$. Observe that there exists a PC-closed, QC closed subset of (the sorts of $L)(($ the formula sort of $\Sigma)$ ) with equality which is $\mathrm{AL}$ closed w.r.t $\varphi$.

An AL theory of $\varphi$ and $L$ is a PC-closed, QC closed, AL closed w.r.t $\varphi$ subset of (the sorts of $L)(($ the formula sort of $\Sigma)$ ) with equality.

\section{Propositional Calculus}

In the sequel $n$ denotes a non empty natural number, $\Sigma$ denotes a non empty, non void, PC correct w.r.t. $n$ PC language signature, $L$ denotes language algebra over $\Sigma, F$ denotes a PC theory of $L$, and $\alpha, \beta, \gamma, \delta$ denote formulae of $L$.

Now we state the propositions:

(35) $\alpha \Rightarrow \alpha \in F$.

(36) $\alpha \wedge \beta \in F$ if and only if $\alpha, \beta \in F$.

(37) $(\alpha \vee \beta) \Rightarrow(\beta \vee \alpha) \in F$.

(38) $(\beta \Rightarrow \gamma) \Rightarrow((\alpha \Rightarrow \beta) \Rightarrow(\alpha \Rightarrow \gamma)) \in F$.

(39) If $\alpha \Rightarrow(\beta \Rightarrow \gamma) \in F$, then $\beta \Rightarrow(\alpha \Rightarrow \gamma) \in F$. The theorem is a consequence of $(38)$.

(40) $(\alpha \Rightarrow \beta) \Rightarrow((\beta \Rightarrow \gamma) \Rightarrow(\alpha \Rightarrow \gamma)) \in F$. The theorem is a consequence of (38) and (39).

(41) $\alpha \Rightarrow(\beta \Rightarrow(\alpha \Rightarrow \beta)) \in F$.

(42) $(\alpha \Rightarrow(\beta \Rightarrow \gamma)) \Rightarrow(\beta \Rightarrow(\alpha \Rightarrow \gamma)) \in F$. The theorem is a consequence of (39) and (38).

(43) $\beta \Rightarrow((\beta \Rightarrow \alpha) \Rightarrow \alpha) \in F$. The theorem is a consequence of (42) and $(35)$.

(44) $\alpha \Leftrightarrow \beta \in F$ if and only if $\alpha \Rightarrow \beta, \beta \Rightarrow \alpha \in F$. The theorem is a consequence of $(36)$.

(45) If $\beta \in F$, then $\alpha \Rightarrow \beta \in F$. 
(46) If $\alpha \Rightarrow \beta, \beta \Rightarrow \gamma \in F$, then $\alpha \Rightarrow \gamma \in F$. The theorem is a consequence of (40).

(47) If $\gamma \Rightarrow(\beta \Rightarrow \alpha), \beta \in F$, then $\gamma \Rightarrow \alpha \in F$. The theorem is a consequence of $(42)$.

(48) $((\alpha \wedge \beta) \Rightarrow \gamma) \Rightarrow(\alpha \Rightarrow(\beta \Rightarrow \gamma)) \in F$. The theorem is a consequence of (45), (40), and (42).

(49) $(\alpha \Rightarrow(\beta \Rightarrow \gamma)) \Rightarrow((\alpha \wedge \beta) \Rightarrow \gamma) \in F$. The theorem is a consequence of (40), (45), (42), (46), and (47).

(50) $(\gamma \Rightarrow \alpha) \Rightarrow((\gamma \Rightarrow \beta) \Rightarrow(\gamma \Rightarrow(\alpha \wedge \beta))) \in F$. The theorem is a consequence of (45) and (46).

(51) $(\alpha \wedge \beta) \Rightarrow(\beta \wedge \alpha) \in F$. The theorem is a consequence of (50).

(52) $(\alpha \Leftrightarrow \beta) \Rightarrow(\beta \Leftrightarrow \alpha) \in F$. The theorem is a consequence of (51), (40), and $(50)$.

(53) $(\alpha \vee \alpha) \Rightarrow \alpha \in F$. The theorem is a consequence of (35).

(54) $\alpha \Rightarrow(\alpha \wedge \alpha) \in F$. The theorem is a consequence of (50) and (35).

(55) If $\alpha \Rightarrow \beta, \alpha \Rightarrow \gamma \in F$, then $\alpha \Rightarrow(\beta \wedge \gamma) \in F$. The theorem is a consequence of (50).

(56) $((\alpha \wedge \beta) \vee(\alpha \wedge \gamma)) \Rightarrow(\alpha \wedge(\beta \vee \gamma)) \in F$. The theorem is a consequence of (46) and (50).

(57) $(\alpha \vee(\beta \wedge \gamma)) \Rightarrow((\alpha \vee \beta) \wedge(\alpha \vee \gamma)) \in F$. The theorem is a consequence of $(50)$ and (46).

(58) $\alpha \Rightarrow(\neg \alpha \Rightarrow \beta) \in F$. The theorem is a consequence of (48).

(59) $(\alpha \Rightarrow \beta) \Rightarrow(\neg \beta \Rightarrow \neg \alpha) \in F$. The theorem is a consequence of (39) and (46).

(60) $\alpha \Rightarrow \beta \in F$ if and only if $\neg \beta \Rightarrow \neg \alpha \in F$. The theorem is a consequence of (59).

(61) If $\alpha \Rightarrow \beta, \gamma \Rightarrow \delta \in F$, then $(\alpha \vee \gamma) \Rightarrow(\beta \vee \delta) \in F$. The theorem is a consequence of (46).

(62) $(\alpha \Rightarrow \beta) \Rightarrow((\gamma \vee \alpha) \Rightarrow(\gamma \vee \beta)) \in F$. The theorem is a consequence of (35), (49), (46), and (48).

(63) If $\alpha \Rightarrow \beta, \gamma \Rightarrow \delta, \neg \beta \vee \neg \delta \in F$, then $\neg \alpha \vee \neg \gamma \in F$. The theorem is a consequence of (59) and (61).

(64) $(\alpha \vee \beta) \Rightarrow(\neg \alpha \Rightarrow \beta) \in F$. The theorem is a consequence of (58).

(65) $(\alpha \vee \beta) \Rightarrow(\neg \beta \Rightarrow \alpha) \in F$. The theorem is a consequence of (37), (64), and (46).

(66) $\alpha \Rightarrow \neg \neg \alpha \in F$. The theorem is a consequence of (35) and (45).

(67) $\neg \neg \alpha \Rightarrow \alpha \in F$. The theorem is a consequence of (66). 
(68) $\alpha \Leftrightarrow \neg \neg \alpha \in F$. The theorem is a consequence of (66), (67), and (36).

(69) $\alpha \Rightarrow \neg \beta \in F$ if and only if $\beta \Rightarrow \neg \alpha \in F$. The theorem is a consequence of (60), (66), and (46).

(70) $\neg \alpha \Rightarrow \beta \in F$ if and only if $\neg \beta \Rightarrow \alpha \in F$. The theorem is a consequence of (60), (67), and (46).

(71) If $\alpha \Rightarrow(\beta \Rightarrow \gamma), \gamma \Rightarrow \delta \in F$, then $\alpha \Rightarrow(\beta \Rightarrow \delta) \in F$. The theorem is a consequence of (49), (46), and (48).

(72) $\neg(\alpha \wedge \beta) \Rightarrow(\neg \alpha \vee \neg \beta) \in F$. The theorem is a consequence of $(60),(67)$, (46), and (50).

(73) $\neg(\alpha \vee \beta) \Rightarrow(\neg \alpha \wedge \neg \beta) \in F$. The theorem is a consequence of (60) and $(50)$.

(74) If $\alpha \Rightarrow \beta, \gamma \Rightarrow \delta \in F$, then $(\alpha \wedge \gamma) \Rightarrow(\beta \wedge \delta) \in F$. The theorem is a consequence of (50) and (46).

(75) $(\neg \alpha \vee \neg \beta) \Rightarrow \neg(\alpha \wedge \beta) \in F$. The theorem is a consequence of (60).

(76) $(\neg \alpha \wedge \neg \beta) \Rightarrow \neg(\alpha \vee \beta) \in F$. The theorem is a consequence of (75), (66), (61), (46), and (60).

(77) $(\alpha \vee(\beta \vee \gamma)) \Rightarrow((\alpha \vee \beta) \vee \gamma) \in F$. The theorem is a consequence of (46), (35), and (61).

(78) $\quad((\alpha \vee \beta) \vee \gamma) \Leftrightarrow(\alpha \vee(\beta \vee \gamma)) \in F$. The theorem is a consequence of (35), (61), (46), (47), and (44).

(79) $(\alpha \wedge(\beta \wedge \gamma)) \Rightarrow((\alpha \wedge \beta) \wedge \gamma) \in F$. The theorem is a consequence of $(50)$, $(35),(74)$, and (46).

(80) $((\alpha \wedge \beta) \wedge \gamma) \Leftrightarrow(\alpha \wedge(\beta \wedge \gamma)) \in F$. The theorem is a consequence of $(50)$, (46), (35), (74), and (44).

(81) $\quad(\gamma \vee(\alpha \Rightarrow \beta)) \Rightarrow((\gamma \vee \alpha) \Rightarrow(\gamma \vee \beta)) \in F$. The theorem is a consequence of (45), (42), and (62).

(82) $\quad((\alpha \vee \beta) \wedge(\alpha \vee \gamma)) \Rightarrow(\alpha \vee(\beta \wedge \gamma)) \in F$. The theorem is a consequence of $(35),(61),(81),(46)$, and (49).

(83) $\quad(\alpha \wedge(\beta \vee \gamma)) \Rightarrow((\alpha \wedge \beta) \vee(\alpha \wedge \gamma)) \in F$. The theorem is a consequence of (82), (72), (74), (73), (46), (35), (76), (75), (61), and (60).

(84) $(\alpha \Rightarrow \beta) \Rightarrow(\neg \alpha \vee \beta) \in F$. The theorem is a consequence of (35), (49), (61), (82), (46), (51), (48), and (37).

(85) $(\alpha \Rightarrow \beta) \Rightarrow \neg(\alpha \wedge \neg \beta) \in F$. The theorem is a consequence of (84), (66), (35), (61), (46), and (75).

(86) $(\beta \vee(\neg \gamma \wedge \gamma)) \Rightarrow \beta \in F$. The theorem is a consequence of (51), (35), (46), (61), and (53). 
(87) $(\beta \vee(\gamma \wedge \neg \gamma)) \Rightarrow \beta \in F$. The theorem is a consequence of (35), (61), $(53)$, and (46).

(88) $\quad(\alpha \Leftrightarrow \beta) \Rightarrow((\alpha \wedge \beta) \vee(\neg \alpha \wedge \neg \beta)) \in F$. The theorem is a consequence of (84), (46), (50), (83), (51), (86), (37), (87), (61), and (35).

(89) $(\alpha \Leftrightarrow \beta) \Rightarrow((\alpha \vee \neg \beta) \wedge(\neg \alpha \vee \beta)) \in F$. The theorem is a consequence of (51), (56), (61), (46), (35), (37), and (88).

(90) $\neg(\alpha \wedge \neg \alpha) \in F$. The theorem is a consequence of (35), (66), (76), (74), (46), (60), and (51).

(91) $\alpha \Leftrightarrow \alpha \in F$. The theorem is a consequence of (35) and (36).

(92) If $\alpha \Leftrightarrow \beta \in F$, then $\beta \Leftrightarrow \alpha \in F$. The theorem is a consequence of (36).

(93) If $\alpha \Leftrightarrow \beta, \beta \Leftrightarrow \gamma \in F$, then $\alpha \Leftrightarrow \gamma \in F$. The theorem is a consequence of (46) and (36).

(94) If $\alpha \Leftrightarrow \beta, \beta \Rightarrow \gamma \in F$, then $\alpha \Rightarrow \gamma \in F$. The theorem is a consequence of $(46)$.

(95) If $\alpha \Rightarrow \beta, \beta \Leftrightarrow \gamma \in F$, then $\alpha \Rightarrow \gamma \in F$. The theorem is a consequence of $(46)$.

(96) $\alpha \Leftrightarrow \beta \in F$ if and only if $\neg \alpha \Leftrightarrow \neg \beta \in F$. The theorem is a consequence of (59) and (36).

(97) $\alpha \Leftrightarrow \beta \in F$ if and only if $\neg \neg \alpha \Leftrightarrow \beta \in F$. The theorem is a consequence of (66), (67), (36), (92), and (93).

(98) If $\alpha \Rightarrow(\beta \Rightarrow \gamma), \delta \Rightarrow \beta \in F$, then $\alpha \Rightarrow(\delta \Rightarrow \gamma) \in F$. The theorem is a consequence of (42) and (46).

(99) If $\alpha \Leftrightarrow(\beta \wedge \gamma), \gamma \Leftrightarrow \delta \in F$, then $\alpha \Leftrightarrow(\beta \wedge \delta) \in F$. The theorem is a consequence of (92), (44), (35), (74), (94), and (95).

(100) If $\alpha \Leftrightarrow(\beta \wedge \gamma), \beta \Leftrightarrow \delta \in F$, then $\alpha \Leftrightarrow(\delta \wedge \gamma) \in F$. The theorem is a consequence of (92), (44), (35), (74), (94), and (95).

(101) If $\alpha \Leftrightarrow(\beta \vee \gamma), \gamma \Leftrightarrow \delta \in F$, then $\alpha \Leftrightarrow(\beta \vee \delta) \in F$. The theorem is a consequence of (92), (44), (35), (61), (94), and (95).

(102) If $\alpha \Leftrightarrow(\beta \vee \gamma), \beta \Leftrightarrow \delta \in F$, then $\alpha \Leftrightarrow(\delta \vee \gamma) \in F$. The theorem is a consequence of (92), (44), (35), (61), (94), and (95).

Let us assume that $\alpha \Rightarrow \beta \in F$. Now we state the propositions:

(103) $(\beta \Rightarrow \gamma) \Rightarrow(\alpha \Rightarrow \gamma) \in F$. The theorem is a consequence of (40).

(104) $(\gamma \Rightarrow \alpha) \Rightarrow(\gamma \Rightarrow \beta) \in F$. The theorem is a consequence of (40) and (39).

Now we state the proposition:

(105) If $\alpha \Rightarrow \beta, \gamma \Rightarrow \delta \in F$, then $(\beta \Rightarrow \gamma) \Rightarrow(\alpha \Rightarrow \delta) \in F$. The theorem is a consequence of (103), (104), and (46). 


\section{Quantifier Calculus}

From now on $\Omega$ denotes a non empty, non void signature, $\mathfrak{T}$ denotes a nonempty algebra over $\Omega, \mathcal{X}$ denotes a non empty yielding generator set of $\mathfrak{T}$, $\Sigma_{1}$ denotes an $\Omega$-extension, non empty, non void, PC correct w.r.t. $n$, QC correct QC language signature over $\bigcup \mathcal{X}, L$ denotes a non-empty language over $\mathcal{X}$ extended by $\left(\emptyset\right.$, (the carrier of $\left.\left.\Sigma_{1}\right)\right)$ and $\Sigma_{1}, G$ denotes a QC theory of $L, \alpha, \beta$, $\gamma, \delta$ denote formulae of $L, x, y, z$ denote elements of $\bigcup \mathcal{X}$, and $x_{0}, y_{0}, z_{0}$ denote elements of $\bigcup\left(\mathcal{X}\right.$ extended by $\left(\emptyset\right.$, (the carrier of $\left.\left.\left.\Sigma_{1}\right)\right)\right)$.

Now we state the propositions:

(106) If $L$ is substitution correct (1), then $\forall x \alpha \Rightarrow \alpha \in G$. The theorem is a consequence of (1), (16), (24), and (14).

(107) $\exists x \alpha \Leftrightarrow \neg \forall x(\neg \alpha) \in G$. The theorem is a consequence of (96) and (97).

(108) $\forall x \alpha \Leftrightarrow \neg \exists_{x}(\neg \alpha) \in G$. The theorem is a consequence of (92), (96), and (97).

(109) If $L$ is substitution correct (1), then $\forall x(\alpha \Rightarrow \beta) \Rightarrow\left(\forall_{x} \alpha \Rightarrow \beta\right) \in G$. The theorem is a consequence of (1), (13), (24), (25), (16), (14), and (98).

(110) Let us consider a sort symbol $a$ of $\Omega$. Suppose $x \in \mathcal{X}(a)$ and $x \notin(\operatorname{vf} \alpha)(a)$ and $\forall x(\alpha \Rightarrow \beta) \in G$. Then $\alpha \Rightarrow \forall x \beta \in G$.

(111) Suppose $L$ is substitution correct (1) and vf-QC-correct. Then $\forall x(\alpha \Rightarrow$ $\beta) \Rightarrow(\forall x \alpha \Rightarrow \forall x \beta) \in G$. The theorem is a consequence of (109), (110), and (46).

(112) Suppose $L$ is substitution correct (1). Let us consider a sort symbol $a$ of $\Omega$. Suppose $x, y \in \mathcal{X}(a)$ and $x_{0}=x$ and $y_{0}=y$. Then $\alpha_{\left(x_{0} \leftarrow y_{0}\right)} \Rightarrow \exists_{x} \alpha \in$ $G$. The theorem is a consequence of (16), (8), (2), (24), (14), (60), (28), (66), (107), and (46).

(113) If $L$ is substitution correct (1) and vf-QC-correct, then $\exists x, y=\Leftrightarrow \neg \forall x, y(\neg \alpha) \in G$. The theorem is a consequence of (107), (68), (92), (96), (93), (44), and (111).

(114) If $L$ is substitution correct (1), then $\alpha \Rightarrow \exists_{x} \alpha \in G$. The theorem is a consequence of (8), (2), and (112).

Let us consider a sort symbol $a$ of $\Sigma_{1}$.

Let us assume that $L$ is vf-QC-correct. Now we state the propositions:

(115) If $x \in \mathcal{X}(a)$, then $x \notin(\operatorname{vf} \forall x \alpha)(a)$.

(116) If $x \in \mathcal{X}(a)$, then $x \notin\left(\operatorname{vf} \exists_{x} \alpha\right)(a)$.

Now we state the proposition:

(117) If $L$ is substitution correct (1) and vf-QC-correct and $\alpha \Rightarrow \beta \in G$, then $\forall x \alpha \Rightarrow \forall x \beta \in G$. The theorem is a consequence of (111). 
Let us assume that $L$ is substitution correct (1) and vf-QC-correct. Now we state the propositions:

(118) $\forall x(\neg \alpha \Rightarrow \neg \beta) \Rightarrow\left(\forall x_{x} \beta \Rightarrow \forall x \alpha\right) \in G$. The theorem is a consequence of (117), (111), and (46).

(119) $\forall x(\alpha \Rightarrow \beta) \Rightarrow(\forall x(\neg \beta) \Rightarrow \forall x(\neg \alpha)) \in G$. The theorem is a consequence of (59), (117), (111), and (46).

Now we state the propositions:

(120) If $L$ is substitution correct (1) and vf-QC-correct and $\alpha \Leftrightarrow \beta \in G$, then $\forall x \alpha \Leftrightarrow \forall x \beta \in G$. The theorem is a consequence of (117), (111), (46), and (50).

(121) $\exists_{x}(\neg \alpha) \Leftrightarrow \neg \forall x \alpha \in G$.

Let us assume that $L$ is substitution correct (1) and vf-QC-correct. Now we state the propositions:

(122) Let us consider a sort symbol $a$ of $\Omega$. Suppose $x \in \mathcal{X}(a)$ and $x \notin(\operatorname{vf} \beta)(a)$. Then $\forall x(\alpha \Rightarrow \beta) \Rightarrow\left(\exists_{x} \alpha \Rightarrow \beta\right) \in G$. The theorem is a consequence of (59), (117), (46), and (71).

(123) $\forall x(\alpha \Rightarrow \beta) \Rightarrow\left(\exists_{x} \alpha \Rightarrow \exists_{x} \beta\right) \in G$. The theorem is a consequence of (119), (59), (46), (44), (66), (60), (67), and (105).

(124) $\forall x(\neg \alpha) \Leftrightarrow \neg \exists_{x} \alpha \in G$. The theorem is a consequence of (66), (123), (67), (44), (93), (92), (96), and (97).

(125) $\forall x, y=\Leftrightarrow \neg \exists x, y(\neg \alpha) \in G$. The theorem is a consequence of (108), (68), (92), (96), (93), (44), and (123).

(126) $\forall x \alpha \Leftrightarrow \forall x(\neg \neg \alpha) \in G$. The theorem is a consequence of (67), (66), (117), and (36).

(127) $\forall x(\alpha \wedge \beta) \Rightarrow(\forall x \alpha \wedge \forall x \beta) \in G$. The theorem is a consequence of (117) and (50).

Now we state the propositions:

(128) If $L$ is vf-QC-correct and substitution correct (1), then $(\forall x \alpha \wedge \forall x \beta) \Rightarrow$ $\forall x(\alpha \wedge \beta) \in G$. The theorem is a consequence of (106), (74), (115), and $(110)$.

(129) If $L$ is substitution correct (1) and vf-QC-correct, then $\left(\forall_{x} \alpha \vee \forall x \beta\right) \Rightarrow$ $\forall x(\alpha \vee \beta) \in G$. The theorem is a consequence of (106), (61), (115), and (110).

(130) If $L$ is substitution correct (1) and vf-QC-correct and $\alpha \Rightarrow \beta \in G$, then $\exists_{x} \alpha \Rightarrow \exists_{x} \beta \in G$. The theorem is a consequence of (59), (117), (107), (94), (92), and (95).

(131) If $L$ is substitution correct (1) and vf-QC-correct and $\alpha \Leftrightarrow \beta \in G$, then $\exists_{x} \alpha \Leftrightarrow \exists_{x} \beta \in G$. The theorem is a consequence of (130) and (36). 
Let us assume that $L$ is substitution correct (1) and vf-QC-correct. Now we state the propositions:

(132) $\exists_{x} \alpha \Leftrightarrow \exists_{x}(\neg \neg \alpha) \in G$. The theorem is a consequence of (68) and (131).

(133) $\left(\exists_{x} \alpha \vee \exists_{x} \beta\right) \Leftrightarrow \exists_{x}(\alpha \vee \beta) \in G$. The theorem is a consequence of (127), (60), (75), (46), (73), (117), (107), (92), (95), (44), (61), (76), (72), and (128).

Now we state the proposition:

(134) Suppose $L$ is substitution correct (1). Let us consider a sort symbol $a$ of $\Omega$. Suppose $x \in \mathcal{X}(a)$ and $x \notin(\operatorname{vf} \alpha)(a)$. Then $\alpha \Leftrightarrow \forall x \alpha \in G$. The theorem is a consequence of (109), (35), (110), and (44).

In the sequel $a$ denotes a sort symbol of $\Omega$.

Let us assume that $L$ is substitution correct (1) and vf-QC-correct and $x \in$ $\mathcal{X}(a)$ and $x \notin(\mathrm{vf} \alpha)(a)$. Now we state the propositions:

(135) $\forall x(\alpha \vee \beta) \Rightarrow(\alpha \vee \forall x \beta) \in G$. The theorem is a consequence of (64), (117), (46), (35), (67), (61), and (84).

(136) $\exists_{x}(\alpha \wedge \beta) \Rightarrow\left(\alpha \wedge \exists_{x} \beta\right) \in G$. The theorem is a consequence of (129), (60), (75), (117), (107), (44), (46), (74), (73), (35), and (122).

(137) $\exists_{x}(\alpha \wedge \beta) \Leftrightarrow\left(\alpha \wedge \exists_{x} \beta\right) \in G$. The theorem is a consequence of (135), (60), (124), (35), (44), (61), (75), (69), (46), (72), (107), (111), (70), and (136).

(138) $\exists_{x}(\alpha \Rightarrow \beta) \Rightarrow\left(\alpha \Rightarrow \exists_{x} \beta\right) \in G$. The theorem is a consequence of $(35)$, (110), (74), (128), (46), (60), (66), (61), (75), (84), (117), (107), (94), (44), (72), (64), and (105).

Now we state the proposition:

(139) If $L$ is vf-QC-correct, then $\forall_{x} \alpha \Rightarrow \forall x, x \alpha \in G$. The theorem is a consequence of (8), (115), (35), and (110).

Let us assume that $L$ is vf-QC-correct and substitution correct (1). Now we state the propositions:

(140) $\forall x, y \alpha \Rightarrow \forall y, x \alpha \in G$. The theorem is a consequence of (106), (111), (115), and (110).

(141) $\exists_{x, y} \alpha \Rightarrow \exists_{y, x} \alpha \in G$. The theorem is a consequence of (140), (60), (113), (92), (94), and (95).

(142) $\exists_{x}\left(\forall_{y} \alpha\right) \Rightarrow \forall_{y}\left(\exists_{x} \alpha\right) \in G$. The theorem is a consequence of (114), (111), (116), and (122).

Let us assume that $L$ is substitution correct (1) and vf-QC-correct. Now we state the propositions:

(143) $\forall x(\alpha \wedge \alpha) \Leftrightarrow \forall x \alpha \in G$. The theorem is a consequence of (54), (111), and (44). 
(144) $\forall x(\alpha \vee \alpha) \Leftrightarrow \forall x \alpha \in G$. The theorem is a consequence of (53), (111), and (44).

(145) $\exists_{x}(\alpha \vee \alpha) \Leftrightarrow \exists_{x} \alpha \in G$. The theorem is a consequence of (53), (44), and (131).

From now on $L$ denotes a non-empty, $\mathfrak{T}$-extension language over $\mathcal{X}$ extended by $\left(\emptyset,\left(\right.\right.$ the carrier of $\left.\left.\Sigma_{1}\right)\right)$ and $\Sigma_{1}, G_{1}$ denotes a QC theory of $L$ with equality, $\alpha, \beta, \gamma, \delta$ denote formulae of $L, \sigma, \sigma_{1}$ denote sort symbols of $\Sigma_{1}, \tau$ denotes elements of $L$ from $\sigma$, and $\tau_{1}, \tau_{2}, \tau_{3}$ denote elements of $L$ from $\sigma_{1}$.

Now we state the proposition:

(146) Suppose $L$ is substitution eq-correct and $x_{0} \in \mathcal{X}(\sigma)$ and $\tau_{1}={ }_{L} \tau_{2} \in G_{1}$. Then $\tau_{1\left(x_{0} \leftarrow \tau\right)}={ }_{L} \tau_{2\left(x_{0} \leftarrow \tau\right)} \in G_{1}$. The theorem is a consequence of (1).

Let us assume that $L$ is substitution eq-correct, vf finite, substitution correct (2), and substitution correct (3) and $\sigma_{1} \in$ the carrier of $\Omega$ and $\mathcal{X}\left(\sigma_{1}\right)$ is infinite. Now we state the propositions:

(147) $\left(\tau_{1}={ }_{L} \tau_{2}\right) \Rightarrow\left(\tau_{2}={ }_{L} \tau_{1}\right) \in G_{1}$. The theorem is a consequence of $(1),(25)$, (16), and (47).

(148) $\quad\left(\left(\tau_{1}={ }_{L} \tau_{2}\right) \wedge\left(\tau_{2}={ }_{L} \tau_{3}\right)\right) \Rightarrow\left(\tau_{1}={ }_{L} \tau_{3}\right) \in G_{1}$. The theorem is a consequence of (1), (25), (16), (147), (46), and (49).

Let us assume that $L$ is substitution correct (3), vf finite, substitution correct (2), substitution correct (1), substitution eq-correct, vf-QC-correct, and vf-eqcorrect and $x=x_{0} \in \mathcal{X}(\sigma)$ and $y=y_{0} \in \mathcal{X}(\sigma)$ and $x \neq y \notin(\operatorname{vf} \alpha)(\sigma)$ and $\mathcal{X}(\sigma)$ is infinite. Now we state the propositions:

(149) $\forall x\left(\alpha \Leftrightarrow \exists_{y}\left(\left(x={ }_{L} y\right) \wedge \alpha_{\left(x_{0} \leftarrow y_{0}\right)}\right)\right) \in G_{1}$. The theorem is a consequence of (16), (147), (46), (1), (24), (14), (49), (122), (112), (28), (35), (45), (55), and (44).

(150) $\forall x\left(\alpha \Leftrightarrow \forall y\left(\left(x={ }_{L} y\right) \Rightarrow \alpha_{\left(x_{0} \leftarrow y_{0}\right)}\right)\right) \in G_{1}$. The theorem is a consequence of (16), (1), (24), (14), (39), (110), (112), (28), (35), (45), (55), (46), (70), (66), (75), (61), (84), (69), (130), (60), (108), (94), and (44).

Now we state the proposition:

(151) Suppose $L$ is substitution correct (1), substitution eq-correct, substitution correct (3), and vf-eq-correct and $x, y \in \mathcal{X}(\sigma)$ and $x \neq y$. Then $\forall x\left(\exists y\left(x={ }_{L} y\right)\right) \in G_{1}$. The theorem is a consequence of $(16),(25),(1),(24)$, (112), and (14).

Let us assume that $L$ is substitution correct (1) and $x=x_{0} \in \mathcal{X}(\sigma)$ and $y=y_{0} \in \mathcal{X}(\sigma)$. Now we state the propositions:

(152) $\left(\alpha \wedge\left(x={ }_{L} y\right)\right) \Rightarrow \alpha_{\left(x_{0} \leftarrow y_{0}\right)} \in G_{1}$. The theorem is a consequence of $(16)$, (1), (24), (14), (39), and (49). 
(153) $\left(\alpha \wedge \neg\left(\alpha_{\left(x_{0} \leftarrow y_{0}\right)}\right)\right) \Rightarrow \neg\left(x={ }_{L} y\right) \in G_{1}$. The theorem is a consequence of (16), (152), (48), (59), (46), and (49).

\section{Algorithmic Logic}

From now on $n$ denotes a non empty, natural number, $\Omega$ denotes a non empty, non void signature, $\mathfrak{T}$ denotes a non-empty free variable algebra over $\Omega$, $\mathcal{X}$ denotes a non-empty generator set of $\mathfrak{T}, \Sigma$ denotes an essential, $\Omega$-extension, non empty, non void, PC correct w.r.t. $n$, QC correct, AL correct w.r.t. $n$ algorithmic language signature of $\bigcup \mathcal{X}, L$ denotes a non empty if-while algebra of $\mathcal{X}$ over $\Sigma, M, M_{1}, M_{2}$ denote algorithms of $L, \alpha, \beta, \gamma, \varphi$ denote formulae of $L, H$ denotes an AL theory of $\varphi$ and $L, a$ denotes a sort symbol of $\Omega, x, y$ denote elements of $\mathcal{X}(a)$, and $\tau$ denotes an element of $\mathfrak{T}$ from $a$.

Now we state the propositions:

(154) $M \cdot((\alpha \wedge \beta) \wedge \gamma) \Leftrightarrow((M \cdot \alpha \wedge(M \cdot \beta)) \wedge(M \cdot \gamma)) \in H$. The theorem is a consequence of (100).

(155) $M \cdot((\alpha \vee \beta) \vee \gamma) \Leftrightarrow((M \cdot \alpha \vee(M \cdot \beta)) \vee(M \cdot \gamma)) \in H$. The theorem is a consequence of (102).

Let us assume that $\alpha \Leftrightarrow \beta \in H$. Now we state the propositions:

(156) $U(M, \alpha) \Leftrightarrow \uplus(M, \beta) \in H$. The theorem is a consequence of (44).

(157) $\cap(M, \alpha) \Leftrightarrow \cap(M, \beta) \in H$. The theorem is a consequence of (44).

Now we state the propositions:

$(158) \cup(M, \alpha) \Leftrightarrow((\alpha \vee(M \cdot \alpha)) \vee \cup(M,(M ; M) \cdot \alpha)) \in H$. The theorem is a consequence of (101), (78), (92), (93), and (156).

(159) $\cap(M, \alpha) \Leftrightarrow((\alpha \wedge(M \cdot \alpha)) \wedge \cap(M,(M ; M) \cdot \alpha)) \in H$. The theorem is a consequence of (99), (80), (92), (93), and (157).

(160) Let us consider elements $x_{0}, y_{0}$ of $\bigcup(\mathcal{X}$ extended by $(\emptyset$, (the carrier of $\Sigma))$ ). Suppose $x=x_{0}$ and $y=y_{0}$. Then $x:={ }_{L}\left({ }^{@} y\right) \cdot \alpha \Leftrightarrow \alpha_{\left(x_{0} \leftarrow y_{0}\right)} \in H$. The theorem is a consequence of (8), (16), (24), and (14).

(161) Suppose $M \cdot \varphi, M \cdot\left(M_{1} \cdot \alpha\right) \in H$ or $M \cdot \neg \varphi, M \cdot\left(M_{2} \cdot \alpha\right) \in H$. Then if $M$ then $M_{1}$ else $M_{2} \cdot \alpha \in H$. The theorem is a consequence of (36) and (44).

(162) Suppose $M \cdot \neg \varphi, \alpha \in H$ or $M \cdot \varphi, M \cdot\left(M_{1} \cdot\left(\right.\right.$ while $\left.\left.M \operatorname{do} M_{1} \cdot \alpha\right)\right) \in H$. Then while $M$ do $M_{1} \cdot \alpha \in H$. The theorem is a consequence of (36) and (44). 


\section{REFERENCES}

[1] Grzegorz Bancerek. Mizar analysis of algorithms: Preliminaries. Formalized Mathematics, 15(3):87-110, 2007. doi 10.2478/v10037-007-0011-x.

[2] Grzegorz Bancerek. Program algebra over an algebra. Formalized Mathematics, 20(4): 309-341, 2012. doi 10.2478/v10037-012-0037-6.

[3] Grzegorz Bancerek. Cardinal numbers Formalized Mathematics, 1(2):377-382, 1990.

[4] Grzegorz Bancerek. König's theorem. Formalized Mathematics, 1(3):589-593, 1990.

[5] Grzegorz Bancerek. Algebra of morphisms Formalized Mathematics, 6(2):303-310, 1997.

[6] Grzegorz Bancerek. Institution of many sorted algebras. Part I: Signature reduct of an algebra Formalized Mathematıcs, 6(2):279-287, 1997.

[7] Grzegorz Bancerek. Translations, endomorphisms, and stable equational theories. Formalized Mathematics, 5(4):553-564, 1996.

[8] Grzegorz Bancerek. The fundamental properties of natural numbers Formalized Mathematics, 1(1):41-46, 1990.

[9] Grzegorz Bancerek. The ordinal numbers. Formalized Mathematics, 1(1):91-96, 1990.

[10] Grzegorz Bancerek. Sequences of ordinal numbers. Formalized Mathematics, 1(2):281290, 1990.

[11] Grzegorz Bancerek. Ordinal arithmetics Formalized Mathematics, 1(3):515-519, 1990.

[12] Grzegorz Bancerek and Krzysztof Hryniewiecki. Segments of natural numbers and finite sequences. Formalized Mathematics, 1(1):107-114, 1990.

[13] Grzegorz Bancerek and Andrzej Trybulec. Miscellaneous facts about functions Formalized Mathematics, 5(4):485-492, 1996.

[14] Ewa Burakowska. Subalgebras of the universal algebra. Lattices of subalgebras Formalized Mathematics, 4(1):23-27, 1993.

[15] Czesław Byliński. Binary operations. Formalized Mathematics, 1(1):175-180, 1990.

[16] Czesław Byliński. Finite sequences and tuples of elements of a non-empty sets. Formalized Mathematics, 1(3):529-536, 1990.

[17] Czesław Byliński. Functions and their basic properties. Formalized Mathematics, 1(1): 55-65, 1990.

[18] Czesław Byliński. Functions from a set to a set Formalized Mathematics, 1(1):153-164, 1990.

[19] Czesław Byliński. The modification of a function by a function and the iteration of the composition of a function. Formalızed Mathematıcs, 1(3):521-527, 1990.

[20] Czesław Byliński. Partial functions. Formalized Mathematics, 1(2):357-367, 1990.

[21] Czesław Byliński. Some basic properties of sets Formalized Mathematics, 1(1):47-53, 1990.

[22] Agata Darmochwał. Finite sets. Formalized Mathematics, 1(1):165-167, 1990.

[23] Jarosław Kotowicz, Beata Madras, and Małgorzata Korolkiewicz. Basic notation of universal algebra Formalized Mathematics, 3(2):251-253, 1992.

[24] Elliott Mendelson. Introduction to Mathematical Logic. Chapman Hall/CRC, 1997. http://books .google.pl/books?id=Z01p4QGspoYC

[25] Grażyna Mirkowska and Andrzej Salwicki. Algorithmic Logic. PWN-Polish Scientific Publisher, 1987.

[26] Beata Perkowska. Free universal algebra construction Formalized Mathematics, 4(1): 115-120, 1993.

[27] Beata Perkowska. Free many sorted universal algebra. Formalized Mathematics, 5(1): 67-74, 1996.

[28] Krzysztof Retel. Properties of first and second order cutting of binary relations Formalized Mathematics, 13(3):361-365, 2005.

[29] Andrzej Trybulec. Binary operations applied to functions Formalized Mathematics, 1 (2):329-334, 1990.

[30] Andrzej Trybulec. Tuples, projections and Cartesian products Formalized Mathematics, 1(1):97-105, 1990.

[31] Andrzej Trybulec. Many sorted algebras. Formalized Mathematics, 5(1):37-42, 1996. 
[32] Andrzej Trybulec. Many sorted sets. Formalized Mathematics, 4(1):15-22, 1993.

[33] Zinaida Trybulec. Properties of subsets Formalized Mathematics, 1(1):67-71, 1990.

[34] Edmund Woronowicz. Many argument relations. Formalized Mathematics, 1(4):733-737, 1990.

[35] Edmund Woronowicz. Relations and their basic properties Formalized Mathematics, 1 (1):73-83, 1990 .

Received September 15, 2014 